\title{
Neural Heterogeneities Determine Response Characteristics to Second-, but Not First-Order Stimulus Features
}

\author{
Michael G. Metzen ${ }^{1}$ and ${ }^{\oplus}$ Maurice J. Chacron ${ }^{1,2}$ \\ 'Department of Physiology and 2Department of Physics, McGill University, Montréal, Québec H3G 1Y6, Canada
}

\begin{abstract}
Neural heterogeneities are seen ubiquitously, but how they determine neural response properties remains unclear. Here we show that heterogeneities can either strongly, or not at all, influence neural responses to a given stimulus feature. Specifically, we recorded from peripheral electroreceptor neurons, which display strong heterogeneities in their resting discharge activity, in response to naturalistic stimuli consisting of a fast time-varying waveform (i.e., first-order) whose amplitude (i.e., second-order or envelope) varied slowly in the weakly electric fish Apteronotus leptorhynchus. Although electroreceptors displayed relatively homogeneous responses to first-order stimulus features, further analysis revealed two subpopulations with similar sensitivities that were excited or inhibited by increases in the envelope, respectively, for stimuli whose frequency content spanned the natural range. We further found that a linear-nonlinear cascade model incorporating the known linear response characteristics to first-order features and a static nonlinearity accurately reproduced experimentally observed responses to both first- and second-order features for all stimuli tested. Importantly, this model correctly predicted that the response magnitude is independent of either the stimulus waveform's or the envelope's frequency content. Further analysis of our model led to the surprising prediction that the mean discharge activity can be used to determine whether a given neuron is excited or inhibited by increases in the envelope. This prediction was validated by our experimental data. Thus, our results provide key insight as to how neural heterogeneities can determine response characteristics to some, but not other, behaviorally relevant stimulus features.
\end{abstract}

Key words: electroreceptor; envelope; neural heterogeneity; weakly electric fish

\section{Introduction}

Understanding the set of transformations by which incoming sensory input leads to behavioral responses (i.e., the neural code) remains a central problem in neuroscience. Strong heterogeneities in neural populations have been experimentally observed throughout the nervous system (Bannister and Larkman, 1995a, b; Goldberg, 2000; Savard et al., 2011) even within the same cell type (Marder and Goaillard, 2006; Schulz et al., 2006). Although much effort has focused on determining the effects of these heterogeneities on population coding (Marsat and Maler, 2010; Padmanabhan and Urban, 2010; Mejias and Longtin, 2012; Tripathy et al., 2013), considerably less effort has focused on uncovering their role in determining neural response to the often-complex features of behaviorally relevant natural stimuli.

Gymnotiform wave-type weakly electric fish sense perturbations of a self-generated electric organ discharge (EOD) through an array of electroreceptors on their skin that project to higher

Received Sept. 23, 2014; revised Dec. 19, 2014; accepted Jan. 10, 2015.

Author contributions: M.G.M. and M.J.C. designed research; M.G.M. performed research; M.G.M. analyzed data; M.G.M. and M.J.C. wrote the paper.

This work was supported by the Canadian Institutes of Health Research and the Fonds de Recherche du QuébecNature et Technologies to M.J.C.

The authors declare no competing financial interests.

Correspondence should be addressed to Dr. Maurice J. Chacron, Department of Physiology, McGill University,

McIntyre Medical Building, Room 1137, 3655 Promenade Sir William Osler, Montréal, Québec H3G 1Y6, Canada. E-mail: maurice.chacron@mcgill.ca.

DOI:10.1523/JNEUROSCI.3946-14.2015

Copyright $\odot 2015$ the authors $\quad 0270-6474 / 15 / 353124-15 \$ 15.00 / 0$ brain areas (Chacron et al., 2011; Marsat et al., 2012; Krahe and Maler, 2014). Natural electrosensory stimuli comprise the sinusoidal perturbations (i.e., beats or first-order) experienced when two or more fish come into proximity whose depth of modulation (i.e., envelope or second-order) depends on the relative distance and orientation between conspecifics (Yu et al., 2012; Fotowat et al., 2013). Although much is known about how firstorder features are processed across successive stages of the electrosensory system as detailed in recent reviews (Chacron et al., 2011; Marsat et al., 2012; Márquez et al., 2013; Krahe and Maler, 2014), the processing of second-order features is not so well understood (Middleton et al., 2006; Savard et al., 2011; Vonderschen and Chacron, 2011; McGillivray et al., 2012; Stamper et al., 2013). Previous studies have shown that there exist large heterogeneities in the baseline activities of these peripheral afferents (i.e., the spiking activity in the presence of the animal's unmodulated EOD) (Xu et al., 1996; Gussin et al., 2007). However, how these afferents respond to envelopes caused by movement and whether heterogeneities influence these both remain poorly understood.

Here we recorded electroreceptor responses to stimuli consisting of a high-frequency noisy time-varying waveform (i.e., first-order) whose amplitude (i.e., envelope or second-order) varied more slowly. We found that afferent sensitivity was independent of both stimulus and envelope frequency content. Surprisingly, although all afferents responded with increased firing rate to the upstrokes of the stimulus, further analysis revealed two 
subpopulations whose responses were either in phase (i.e., ON) or out of phase (i.e., OFF) with the envelope. A linear-nonlinear cascade model incorporating response properties to the stimulus waveform along with static nonlinearities explained our experimental data and furthermore predicted how baseline firing frequency can determine whether a given afferent's response to the envelope is ON- or OFF-type. Our results thus demonstrate that neural heterogeneities can have little or significant influence on neural response properties depending on different stimulus attributes. We propose that heterogeneities help optimize afferent responses to the largely independent first and second features of electrosensory stimuli.

\section{Materials and Methods}

McGill University's animal care committee approved all experimental procedures.

Animals and recording. We used the gymnotiform wave-type weakly electric fish species Apteronotus leptorhynchus. Animals of either sex were acquired from local tropical fish suppliers and were housed in groups $(2-10)$ at controlled water temperature $\left(26^{\circ} \mathrm{C}-29^{\circ} \mathrm{C}\right)$ and conductivity (300-800 $\mu \mathrm{S} / \mathrm{cm}$ ) according to published guidelines (Hitschfeld et al., 2009). Before surgery, animals were paralyzed with an intramuscular injection of tubocurarine chloride hydrate ( $1 \mu \mathrm{g} / \mathrm{g}$ body weight; Sigma). Surgical methods have been previously described in detail (Bastian, 1996a, b, Bastian et al., 2002; Chacron and Bastian, 2008; Krahe et al., 2008; Toporikova and Chacron, 2009; McGillivray et al., 2012; Simmonds and Chacron, 2015). In brief, animals were transferred to an experimental tank $(30 \mathrm{~cm} \times 30 \mathrm{~cm} \times 10 \mathrm{~cm})$ containing water from the animal's home tank and respired by a constant flow of oxygenated water through their mouth. The animal's head was then locally anesthetized with lidocaine ointment (5\%; AstraZeneca). Subsequently, the skull was partly exposed and a small window was opened over the recording region.

Sharp glass micropipette electrodes $(20-40 \mathrm{M} \Omega)$ backfilled with $3 \mathrm{M}$ $\mathrm{KCl}$ were used to record in vivo from P-type electrosensory afferent axons (P-units) in the deep fiber layer of the electrosensory lateral line lobe (ELL) as described in previous studies (Bastian, 1981; Chacron et al., 2005). The recording electrode was advanced into the ELL with a piezoelectric microdrive (Inchworm, IW-711; Kopf). The recorded potentials were amplified (Axoclamp 2B, Molecular Devices), digitized at $10 \mathrm{kHz}$ sampling rate using CED 1401plus hardware and Spike2 software (Cambridge Electronic Design), and stored on a computer hard disc for offline analysis. The EOD was recorded between the head and tail of the fish by using two vertical metal wires (see Fig. $1 A$, E1 and E2), amplified (model 1700 amplifier, A-M Systems, bandpass filter between $300 \mathrm{~Hz}$ and $5 \mathrm{kHz}$ ) and digitized at $10 \mathrm{kHz}$ using a CED Power1401 with Spike2 software (Cambridge Electronic Design).

Stimulation. It is important to note that the high-frequency $(600-1000$ $\mathrm{Hz}$ ) quasi-sinusoidal EOD waveform generated by the animal can be considered as a carrier signal. As P-units only respond to changes in EOD amplitude (Scheich et al., 1973), the EOD amplitude modulation (AM) is the meaningful stimulus here. For this reason, we will refer to the EOD AM waveform as the stimulus $S(t)$. We will consider both first- and second-order attributes of $S(t)$ as described below, and these correspond to the second-and third-order attributes of the full signal received by the fish, respectively.

When two conspecifics are in close proximity, interference between their EODs creates an EOD AM (i.e., first-order) whose frequency is equal to the difference between the EOD frequencies of both fish and whose amplitude (i.e., the envelope or second-order) changes as a function of the relative distance between both fish (Yu et al., 2012; Fotowat et al., 2013; Metzen and Chacron, 2014). To mimic the small changes in AM frequency observed during natural behaviors (Stamper et al., 2010, 2012; Metzen and Chacron, 2014), we used both low (fourth-order Butterworth, $5-15 \mathrm{~Hz}$ band) and high (fourth-order Butterworth, $60-80 \mathrm{~Hz}$ band) frequency noisy AMs. The AM amplitude (i.e., the envelope) was either constant (i.e., unmodulated) and was set at $\sim 40 \%$ of the baseline EOD amplitude as done previously (Gussin et al., 2007; Savard et al., 2011) or was modulated at $70 \%$ depth around this constant value sinu- soidally at frequencies between 0.05 and $10 \mathrm{~Hz}$ that span the range seen under natural conditions (Yu et al., 2012; Fotowat et al., 2013; Metzen and Chacron, 2014). We ensured that the envelope frequency was always lower than the lowest frequency contained in the AM. Thus, for example, for a $5-15 \mathrm{~Hz} \mathrm{AM}$, we used envelope frequencies of $0.05,0.1,0.2,0.5$, 0.75 , and $1 \mathrm{~Hz}$, whereas, for a $60-80 \mathrm{~Hz} \mathrm{AM}$, we used envelope frequencies of $0.05,0.1,0.2,0.5,0.75,1,5$, and $10 \mathrm{~Hz}$. Additionally, to test the effects of AM frequency content on envelope coding, we used several AMs (fourth-order Butterworth) whose frequency bands spanned the natural range as they were $5-15,15-30,30-45,45-60,60-80,80-100$, and $100-120 \mathrm{~Hz}$ and whose amplitudes were modulated sinusoidally at 0.05 and $0.5 \mathrm{~Hz}$.

Each stimulus $S(t)$ lasted $50 \mathrm{~s}$ and was delivered as amplitude modulation of the EOD by multiplying it (MT3 multiplier, Tucker-Davis Technologies) with a sinusoidal signal that is phase-locked to the animal's own EOD before being attenuated (Leader, LAT-45, Leader Electronics), isolated from ground (World Precision Instruments A395 linear stimulus isolator), and delivered to the experimental tank via a pair of silversilver chloride electrodes located $\sim 20 \mathrm{~cm}$ on each side of the animal. Thus, changes in the animal's EOD frequency will not alter the stimulus $S(t)$ as well as its envelope. This stimulation configuration is referred to as "global" in previous studies because the electric image caused by the stimulus covers most, if not all, of the animal's skin surface (Chacron et al., 2003; Krahe et al., 2008; Toporikova and Chacron, 2009; AvilaAkerberg et al., 2010). We measured the resulting perturbations of the animal's EOD using a small dipole positioned at the center and lateral to the animal 1-2 mm away from the skin (Bastian et al., 2002). Before stimulation, each afferent's baseline activity (i.e., discharge in the presence of the animal's unmodulated EOD) was recorded for a minimum of 20 s. Figure $1 A$ summarizes the different signals and their respective frequency contents.

Data analysis. All analysis was performed offline using custom written routines in MATLAB (MathWorks). Values are reported as mean \pm SEM throughout.

Extracting the stimulus envelope. The envelope can be regarded as the instantaneous amplitude of the stimulus $S(t)$ or its time-varying contrast. It can be obtained from the stimulus $S(t)$ by the following nonlinear transformation:

$$
E(t)=\sqrt{S(t)^{2}+\hat{S}(t)^{2}}
$$

where $\hat{S}(t)$ is the Hilbert transform of $S(t)$ given by (Myers et al., 2003; Middleton et al., 2006; Savard et al., 2011) the following:

$$
\hat{S}(t)=\frac{1}{\pi} C\left[\int_{-\infty}^{+\infty} \frac{S(\tau)}{t-\tau} d \tau\right]
$$

where $C$ is the Cauchy principal value.

Behavior. We quantified the behavioral responses of each fish to the envelope by calculating the parameters gain and phase to each particular envelope frequency using systems identification methods as done in a previous study (Metzen and Chacron, 2014). However, Metzen and Chacron (2014) used sinusoidal AM stimuli that are different than the previously described noisy AMs used here. Briefly, we extracted the time-dependent EOD frequency from the recording to obtain the response $R_{\text {behavior }}(t)$. To compute the gain and the phase, we next averaged the time-varying EOD frequency over the number of envelope cycles present in the stimulus $S(t)$ to get the response $R_{\text {behavior }}$. The gain was calculated as follows:

$$
\text { gain }=\frac{A_{\text {response }}}{A_{\text {envelope }}}
$$

where $A_{\text {response }}$ is the amplitude of the averaged response $R_{\text {behavior }}$, and $A_{\text {envelope }}$ is the amplitude of the envelope $E(t)$.

The phase shift was determined as follows:

$$
\theta=\frac{T_{\max (E)}-T_{\max (R)}}{T_{E}} \times 2 \pi
$$


where $T_{\max (E)}$ and $T_{\max (R)}$ are the times at which the envelope $E$ and the response $R_{\text {behavior }}$ reach their maximum values, respectively.

Electrophysiology. The recorded membrane potential was first highpass filtered (100 Hz; eighth order Butterworth). Spike times were defined as the times at which this signal crossed a given threshold value from below. A binary sequence $R(t)$ was then constructed from the spike times in the following manner: time was first discretized into bins of width $d t=0.1 \mathrm{~ms}$. The value of bin $i$ was set to 1 if there was a spike at time $t_{j}$ such that $\mathrm{i}^{\star} d t<t_{j}<(\mathrm{i}+1){ }^{\star} d t$ and to 0 otherwise. Because the bin width $d t$ is smaller than the absolute refractory period of the neuron, there can be at most one spike time that can occur within any given bin.

To quantify the relationship between the stimulus $S(t)$ and the binary sequence $R(t)$, we used a Kaiser filter as was done previously (Cherif et al., 2008 ) to filter the binary sequence and obtain an estimate of the instantaneous firing rate. The cutoff frequency was set to be $0.1 \%$ higher than the highest frequency of the AM stimulus $S(t)$.

To quantify the relationship between the envelope $E(t)$ and the neural response, we again used a Kaiser filter to filter the binary sequence and obtain an estimate of the time-dependent firing rate. The cutoff frequency here was set to be $0.1 \%$ higher than the envelope frequency. Thus, the cutoff frequency used for envelope stimuli was always lower than that used for AM stimuli, and the fast fluctuations caused by the stimulus waveform will average out when considering the slower timescale at which the envelope varies. The relationship between the envelope $E(t)$ and the firing rate estimate was described using linear-systems identification techniques. The gain and phase were obtained using the formulas described above (Eqs. 3 and 4) for behavior.

Resting discharge. We quantified the resting discharge variability using the coefficient of variation $(\mathrm{CV})$ during baseline activity as follows:

$$
C V=\frac{\operatorname{std}(I S I)}{\operatorname{mean}(I S I)}
$$

where $s t d$ is the standard deviation and ISI is the interspike interval (i.e., the time between consecutive action potentials) sequence. The baseline firing rate was computed as the number of action potentials per unit time that occurred during baseline activity. Overall, we found that the baseline firing rate was nearly constant for each afferent during the recording as estimates of baseline firing rate just before the onset of each stimulus only differed by $0.32 \pm 0.23 \%$ ( $t$ test, $n=414, p=0.89$ ) on average. The normalized baseline firing rate was obtained by dividing the baseline firing rate preceding the onset of each envelope stimulus by the maximum firing rate in response to unmodulated noisy AM stimuli. P-units whose normalized baseline firing rate was not significantly different than 0.5 as determined by a one-sided $t$ test at the $p=0.01$ level were excluded from the relevant analyses as we then could not reliably determine whether the normalized baseline firing rate was less or greater than 0.5 to test modeling predictions.

Modeling. We fit a linear-nonlinear cascade model (Chichilnisky, 2001; Massot et al., 2012) to each primary afferent response in the following way: First, we recorded baseline activity for $20 \mathrm{~s}$ to measure the neuron's mean firing rate. Then, AM stimuli with constant amplitude (i.e., envelope) and with different frequency bands as described above were presented. We then estimated the linear filter $H(t)$ from these data in the following way. Because the AM stimuli used in this study had relatively narrow frequency ranges, we assumed that the gain $|\tilde{H}(f)|$, where $\tilde{H}(f)$ is the Fourier transform of $H(t)$, and the phase $\phi(f)=\arctan (\operatorname{Im}(\tilde{H}(f)) / \operatorname{Re}(\tilde{H}(f)))$, where $\operatorname{Im}(\ldots)$ and $\operatorname{Re}(\ldots)$ denote the imaginary and real parts, respectively, are constant over each AM stimulus' frequency content. The phase was obtained from the time lag at which the autocorrelation function between the stimulus and firing rate was maximal (Massot et al., 2011). This value was then used to align the stimulus and firing rate responses in time. Plotting the mean firing rate response as a function of the stimulus using bins of $0.005 \mathrm{mV} / \mathrm{cm}$ gave rise to a curve that was fitted using a piecewise linear function. We typically used stimulus values $\sim \pm 10 \%$ of the baseline EOD amplitude and performed a linear least-squares fit. We then implemented the piecewise nonlinearity by setting negative values of the fitted firing rate to zero and by setting values greater than the maximum firing rate $\mathrm{FR}_{\max }$ to $\mathrm{FR}_{\max }$.
The gain was calculated from the slope of the linear least-squares fit. Although we used a piecewise linear function to be consistent with previous studies (Nelson et al., 1997; Gussin et al., 2007), quantitatively similar results were obtained when we instead fit a sigmoid function (data not shown).

$H(t)$ was then used to filter the stimulus to obtain the linear prediction to which the baseline firing rate was added. The resulting signal was then passed through a static nonlinearity that takes into account rectification (the firing rate cannot be negative) and saturation (the firing rate cannot be greater than the maximum value $\mathrm{FR}_{\max }$ as determined above) to obtain the predicted firing rate response to stimuli consisting of independent and identically distributed AM waveforms whose envelope was modulated sinusoidally. We first used the model to predict the response to the stimulus waveform (first-order). To predict the firing rate response to the envelope (i.e., second-order), we low-pass filtered the predicted firing rate using a Kaiser filter whose cutoff frequency here was set to be $0.1 \%$ higher than the envelope frequency as done for the experimental data.

Stimulus reconstruction and coding fraction. We used the stimulus reconstruction technique to estimate the time-varying envelope $E(t)$ of our noisy stimulus from a population of $n \mathrm{P}$-units using their spike trains $R_{i}(t)$ (the index $i$ refers to the $i^{\text {th }}$ neuron) recorded during stimulation. Although the spike trains used were not recorded from simultaneously, we note that previous studies have found that $\mathrm{P}$-units displayed negligible noise correlations (Chacron et al., 2005). This approach assumes that the time-varying envelope $E(t)$ can be estimated by convolving each spike train $R_{i}(t)$ with a separate kernel $K_{i}(t)$ and then summing the respective contributions (Warland et al., 1997; Dan et al., 1998; Krahe et al., 2002; Massot et al., 2011) as follows:

$$
E_{e s t}(t)=\sum_{i=1}^{n} \int d \tau K_{i}(\tau) R_{i}(1-\tau)
$$

The optimal set of kernels $K_{i}(\tau)$ that minimizes the mean-square error $\varepsilon^{2}=\left\langle\left[E(t)-E_{\text {est }}(t)\right]^{2}>\right.$ is a solution to the following system of equations (Dayan and Abbott, 2001):

$$
\begin{aligned}
\left(\begin{array}{cccc}
P_{R_{1} R_{1}}(f) & P_{R_{1} R_{2}}(f) & \ldots & P_{R_{1} R_{j}}(f) \\
P_{R_{2} R_{1}}(f) & P_{R_{2} R_{2}}(f) & \ldots & P_{R_{2} R_{j}}(f) \\
\vdots & \vdots & \ddots & \vdots \\
P_{R_{i} R_{1}}(f) & P_{R_{i} R_{2}}(f) & \ldots & P_{R_{i} R_{j}}(f)
\end{array}\right)\left(\begin{array}{c}
\tilde{K}_{1}(f) \\
\tilde{K}_{2}(f) \\
\vdots \\
\tilde{K}_{i}(f)
\end{array}\right) \\
=\left(\begin{array}{c}
P_{E R_{1}}(-f) \\
P_{E R_{2}}(-f) \\
\vdots \\
P_{E R_{i}}(-f)
\end{array}\right)
\end{aligned}
$$

where $\tilde{K}_{i}(f)$ is the Fourier transform of $K_{i}(t)$, and $P_{R i R j}(f)$ is the crossspectrum between binary sequences $R_{i}(t)$ and $R_{j}(t)$. We assessed the quality of linear stimulus reconstruction by computing the coding fraction (Gabbiani et al., 1996; Rieke et al., 1996) as follows:

$$
C F=1-\frac{\varepsilon}{\sigma}
$$

where $\sigma$ is the standard deviation of the envelope $E(t)$. The CF ranges between 0 and 1 and represents the fraction of the stimulus that is correctly estimated (Gabbiani, 1996).

We extrapolated the coding fraction as a function of population size using a nonlinear least squares fit with the following function based on theoretical considerations (Zohary et al., 1994; Gabbiani, 1996) as follows:

$$
\mathrm{f}(\mathrm{n})=1-(\alpha \sqrt{n}) / \sqrt{\mathrm{n}+\mathrm{n}(\mathrm{n}-1) \mathrm{r}}
$$

where $\alpha$ and $\mathrm{r}$ are parameters and $n$ is the population size. This equation was used to calculate the population size needed to reach a coding fraction of 0.8 . 
A
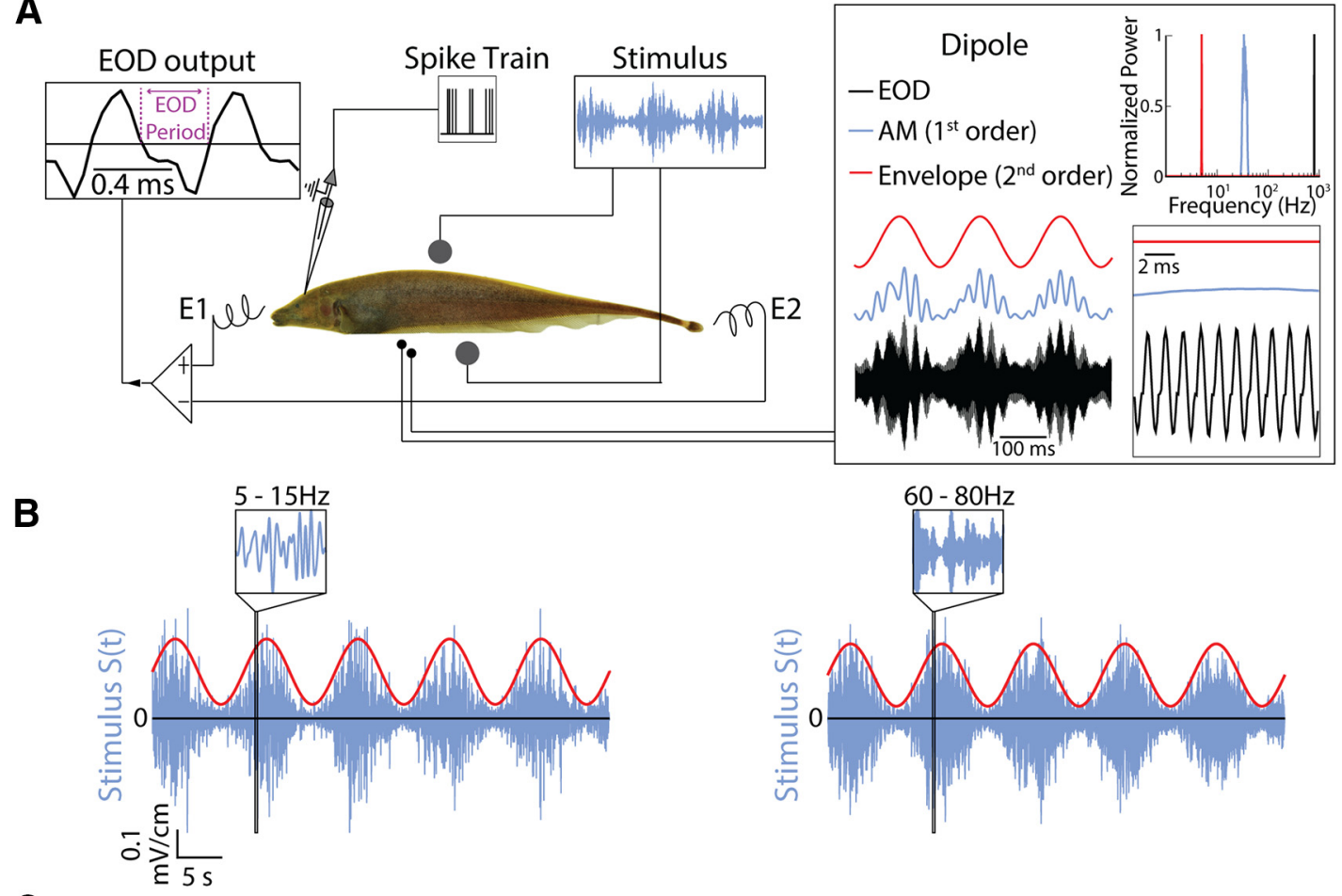

c
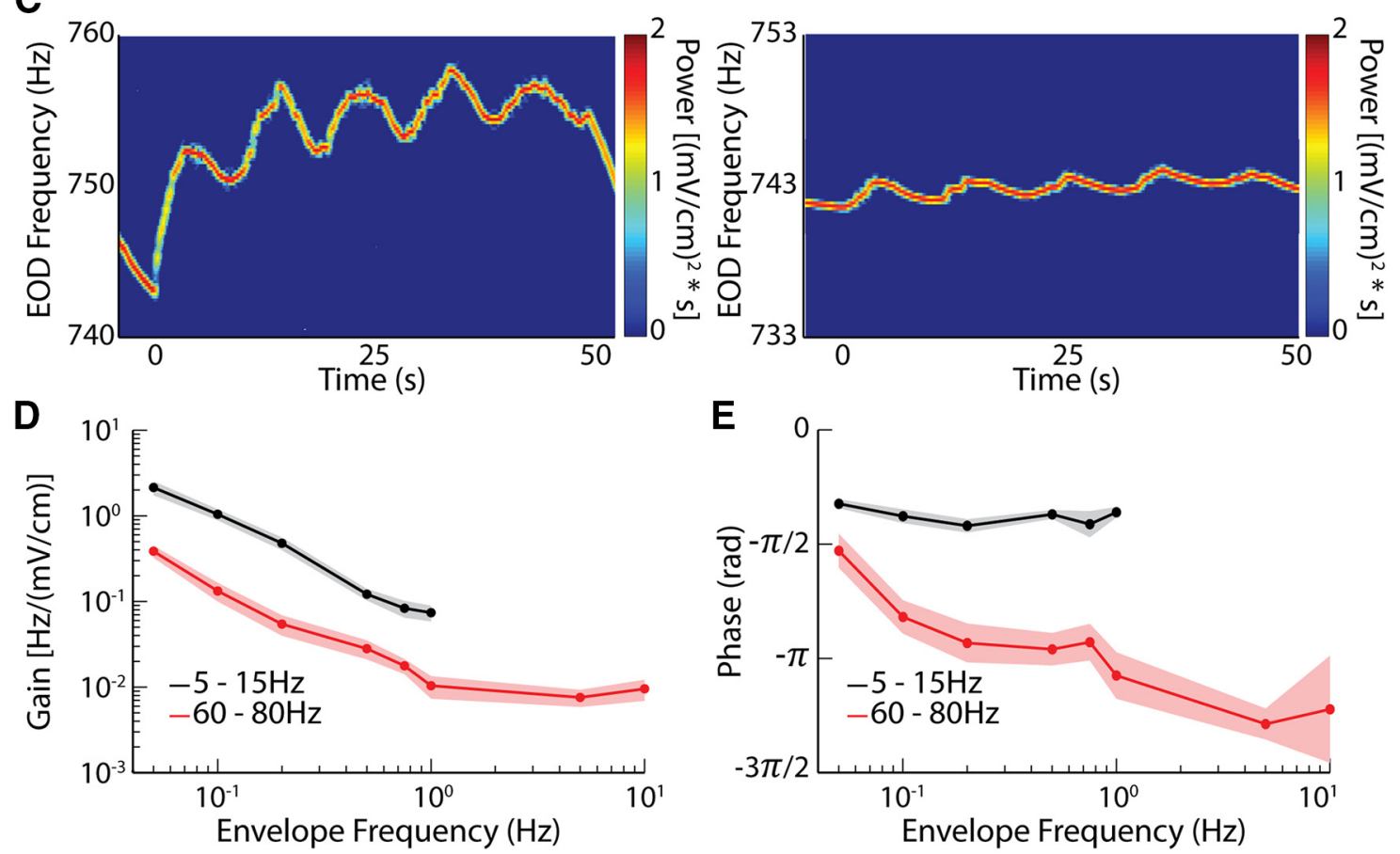

Figure 1. A. leptorhynchus give behavioral responses to envelopes. $A$, Schematic of the experimental setup. The animal's electric field is monitored by a pair of electrodes located in front and behind the animal (E1 and E2) while the stimulus is delivered using a separate set of electrodes positioned on each side (gray spheres). The full signal (inset, black) received by the animal was recorded with a dipole $\sim 2 \mathrm{~mm}$ away from the animal (black spheres). Also shown are the frequency contents of the full signal (black), the noisy AM (blue), and the envelope (red). $\boldsymbol{B}$, Example stimuli showing the stimulus (blue) and its envelope (red) for the two different AM signals used. Left, Frequency range between 5 and $15 \mathrm{~Hz}$, mimicking same sex encounters. Right, Frequency range between 60 and $80 \mathrm{~Hz}$, mimicking opposite sex encounters. Insets, Magnification of the stimulus. C, EOD spectrograms (i.e., EOD power spectrum as a function of time) showing behavioral responses to the stimuli shown in $\boldsymbol{B}$ from an example specimen. $\boldsymbol{D}$, Population-averaged gain as a function of envelope frequency for the two noisy $\mathrm{AM}$ signals used: black represents frequency range $5-15 \mathrm{~Hz}$, $n>10$ for each data point; red represents frequency range $60-80 \mathrm{~Hz}, n>10$ for each data point. $E$, Population-averaged phase as a function of envelope frequency for the two AM signals used: black represents frequency range $5-15 \mathrm{~Hz}, n>10$ for each data point; red represents frequency range $60-80 \mathrm{~Hz}, n>10$ for each data point. Error bars indicate SEM.

\section{Results}

We recorded the electrophysiological responses of peripheral P-type electroreceptor neurons (i.e., P-units) as well as the animal's EOD (Fig. 1A) in response to stimuli consisting of EOD
AMs representing two different behavioral contexts. The EOD AM consisted of bandpass filtered white noise with zero mean that contained low $(5-15 \mathrm{~Hz})$ and high $(60-80 \mathrm{~Hz})$ frequencies (Fig. $1 B$ ) to mimic natural stimuli that arise during encounters 
between same and opposite-sex conspecifics, respectively (Zakon et al., 2002; Fotowat et al., 2013; Metzen and Chacron, 2014). The AM amplitude (i.e., the envelope) varied sinusoidally with frequencies spanning that found under natural conditions (0.05-10 $\mathrm{Hz}$ ) (Yu et al., 2012; Fotowat et al., 2013; Metzen and Chacron, 2014). Figure $1 A$ shows examples of the noisy AM (blue), its envelope (red), and the full signal received by the animal (black) as well as their respective frequency content. It is further important to realize that the animal's EOD is a carrier and that the meaningful stimulus here is the EOD AM. We will be considering both first- and second-order features of the stimulus and note that these correspond to the second- and third-order features of the full signal received by the animal, respectively. We note that natural stimuli in other systems (e.g., auditory, somatosensory) frequently consist of a fast time-varying waveform whose amplitude (e.g., envelope) varies more slowly similar to the stimuli considered here (Baker, 1999; Heil, 2003; Zeng et al., 2005; Lundstrom et al., 2010).

\section{A. leptorhynchus display behavioral responses to envelopes arising from different contexts}

The stimuli used in this study are behaviorally relevant to the animal. Indeed, we found that the animal's EOD frequency followed the detailed time course of the envelope waveform (Fig. $1 C)$. We also observed a stronger behavioral response when the AM stimulus contained low frequencies as seen predominantly during interactions between same-sex conspecifics (Zakon et al., 2002), suggesting that this behavior might serve to deter aggression (Metzen and Chacron, 2014). We used linear-systems identification techniques and computed the gain (i.e., the ratio of output peak-to-peak amplitude in EOD frequency to the envelope peak-to-peak amplitude) as well as the phase (i.e., the amount of time normalized by the stimulus period that the EOD frequency must be shifted to be aligned with the envelope) as a function of envelope frequency. We found that, for a given envelope frequency, the gain was higher for lower AM frequencies. Strikingly, the gain decreased as a power law whose exponent was approximately the same for both low and high-frequency AMs (5-15 Hz: $\alpha=1.19 \pm 0.10 ; 60-80 \mathrm{~Hz}: \alpha=1.12 \pm 0.14$; Fig. $1 D$, compare black and red curves). We also observed a phase lag that was almost constant as a function of envelope frequency when the AM contained low frequencies (Fig. 1E, black curve) and that increased with increasing envelope frequency when the AM contained high frequencies (Fig. $1 E$, red curve).

\section{P-units display heterogeneous responses to envelope stimuli}

Next, we recorded the activity of $n=136 \mathrm{P}$-units from a total of 16 fish under baseline conditions (i.e., in the presence of the animal's unmodulated EOD) and in response to the stimuli described above. We observed strong heterogeneities in baseline activity. For example, the baseline firing frequency ranged between $156 \mathrm{~Hz}$ and $606 \mathrm{~Hz}(322 \pm 103 \mathrm{~Hz})$, and the coefficient of variation of the baseline activity ranged between 0.33 and 0.98 $(0.58 \pm 0.15)$, which is consistent with previous studies (Xu et al., 1996; Chacron et al., 2005; Gussin et al., 2007).

Analysis of $\mathrm{P}$-unit responses to envelope stimuli revealed two distinct classes. One afferent class $(n=81)$ responded to increases in the envelope with increased activity (i.e., ON-type) (Fig. 2A), whereas the other class $(n=55)$ instead responded with decreased activity (i.e., OFF-type) (Fig. $2 B$ ). It is important to note that, henceforth, ON- and OFF-type will only be used to describe responses to envelopes. Strikingly, similar responses were obtained for a given envelope when we used either low or high-frequency AM waveforms (Fig. 2A, $B$, middle, compare black and purple traces). Furthermore, ON- and OFF-type responses were observed for all envelope frequencies tested and the responses largely overlapped when time was normalized with respect to the envelope period (Fig. $2 A, B$, bottom).

Analysis of our dataset revealed that the sensitivities (i.e., gain) of both classes to the envelope were not significantly different from one another for all envelope frequencies and for all AM stimuli used (two-way ANOVA, $p>0.28$; Fig. 2C,D). Responses of ON-type P-units were in phase with the envelope for all envelope and AM frequencies tested independently of the AM's frequency content (Fig. 2E,F, solid black and purple circles). In contrast, responses of OFF-type P-units were out of phase with the envelope for all envelope and AM frequencies tested (Fig. $2 E, F$, open black and purple circles). There were no significant differences between the distributions of gain values for $\mathrm{ON}$ and OFF P-units (Kolmogorov-Smirnov test, $p=0.34$ ) (Fig. $2 G$ ). In contrast, the distribution of phase values were clearly bimodal (Hartigan's dip test, $p \ll 10^{-3}$ ) and ON and OFF P-units displayed phase distributions that were significantly different from one another (Kolmogorov-Smirnov test, $p \ll 10^{-3}$ ) (Fig. $2 H$ ). Our results show that the $\mathrm{P}$-unit population displays strong heterogeneities in responses to envelopes for a wide range of envelope and AM frequencies.

\section{P-units display homogeneous responses to AM stimuli}

Why do P-units display such heterogeneities in response to envelopes? We first tested whether such heterogeneities might be caused by heterogeneous responses to the AM stimulus waveforms that we used in this study. Thus, we quantified P-unit responses to AM stimuli characterized by constant envelopes.

The response from a typical ON-type P-unit to an AM stimulus characterized by a constant envelope consisting of bandpass filtered $(60-80 \mathrm{~Hz})$ white noise is shown in Figure $3 \mathrm{~A}$. We found that, as the spiking activity only occurred during the stimulus upstrokes, the firing rate response led the stimulus (Fig. 3A). Aligning the firing rate response to the stimulus waveform and plotting one versus the other revealed an approximately piecewise linear tuning curve consisting of rectification (i.e., zero firing rate) for strongly negative values, a region where the firing rate increases linearly with the stimulus for values $\sim 0$, and saturation (i.e., firing rate equal to the inverse absolute refractory period) for strongly positive values (Fig. 3B). These responses agree with those found previously using different AM stimuli (Nelson et al., 1997; Gussin et al., 2007). For comparison, the response of a typical OFF-type P-unit to the same unmodulated noise stimulus is shown in Figure $3 C$. This unit's activity also only occurred during the stimulus upstrokes, and its firing rate response also led the stimulus waveform (Fig. 3C). Aligning the firing rate response to the stimulus waveform and plotting one as a function of the other also revealed an approximately piecewise linear tuning curve that was strikingly similar to that obtained for the ON-type P-unit (compare Fig. $3 B, D$ ).

Quantifying ON- and OFF-type P-unit responses to unmodulated (i.e., with constant envelope) AM stimuli consisting of bandpass filtered white noise revealed surprising similarities. Indeed, the gain (i.e., the non-zero slope of the portion of the tuning curve where firing rate and stimulus are linearly related) (Fig. $3 E$ ) and the phase (Fig. $3 F$ ) were not significantly different between both afferent classes (gain: two-way ANOVA, $p>0.08$; phase: two-way ANOVA, $p>0.06$ ). We note that the modulations in firing rates elicited by AM stimuli were significantly larger than 
A
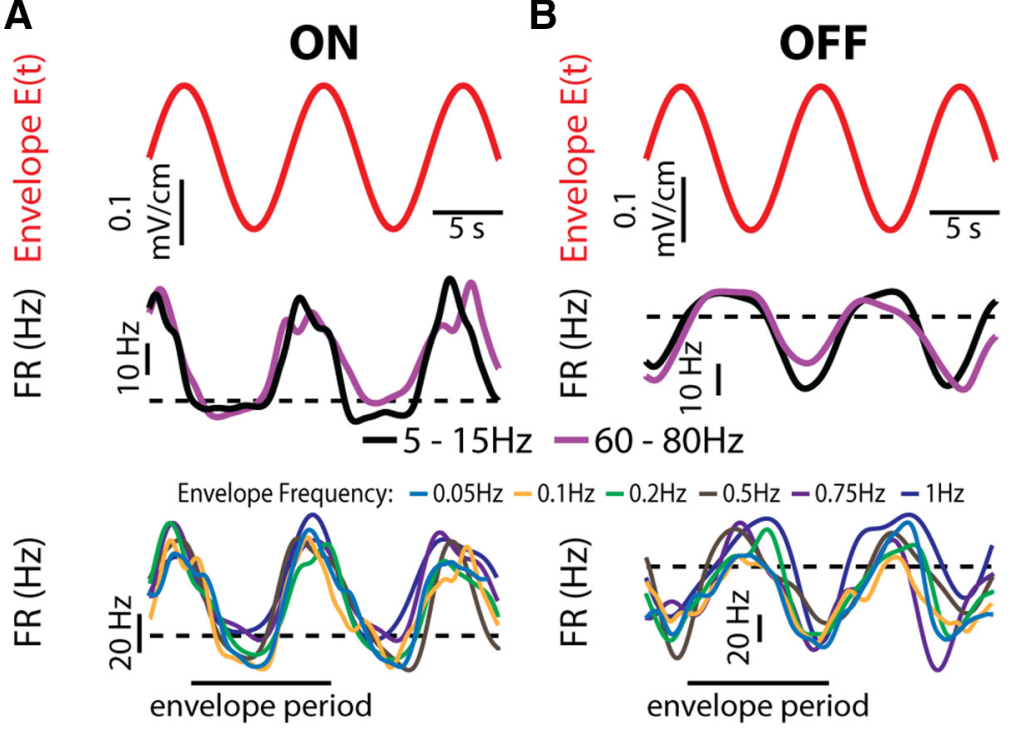

C

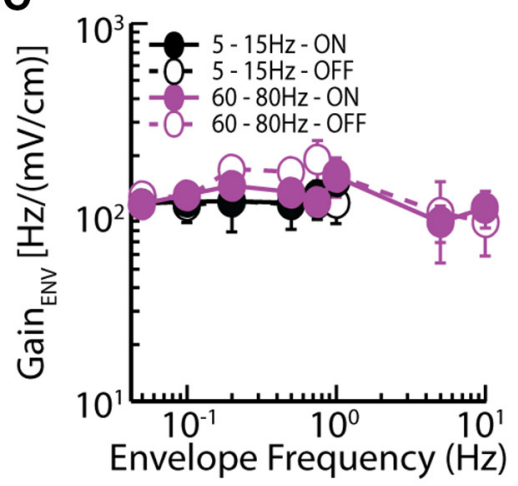

E

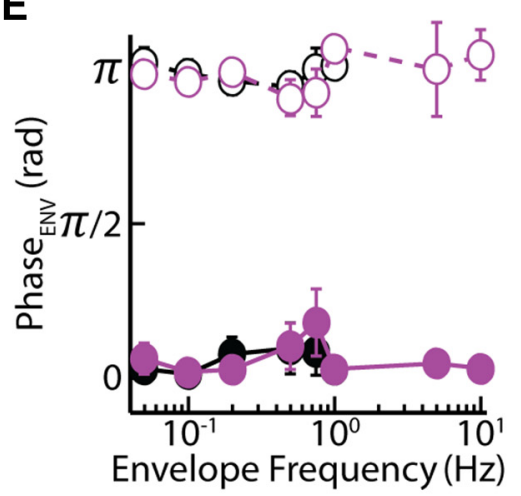

G

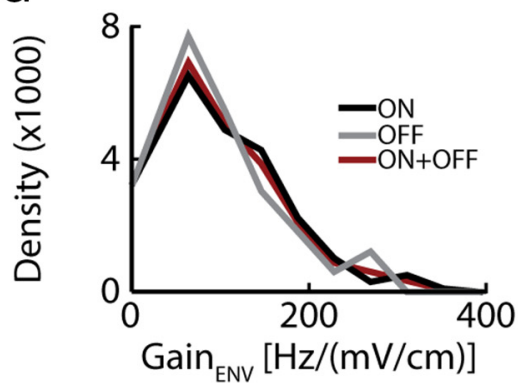

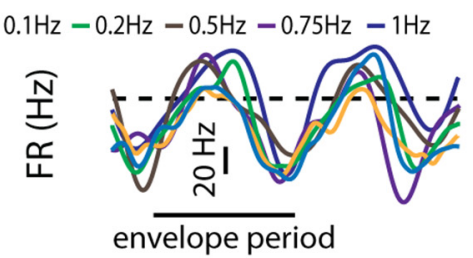

D

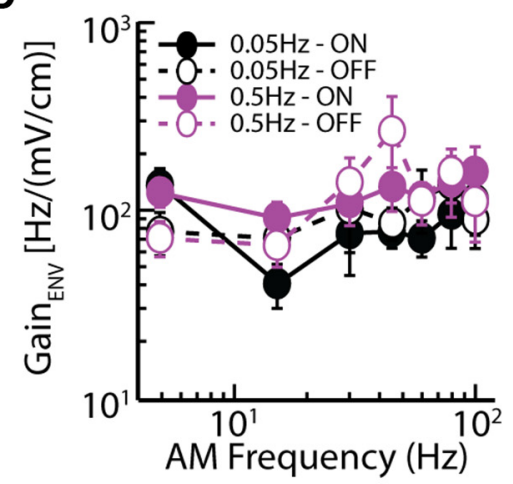

$\mathbf{F}$

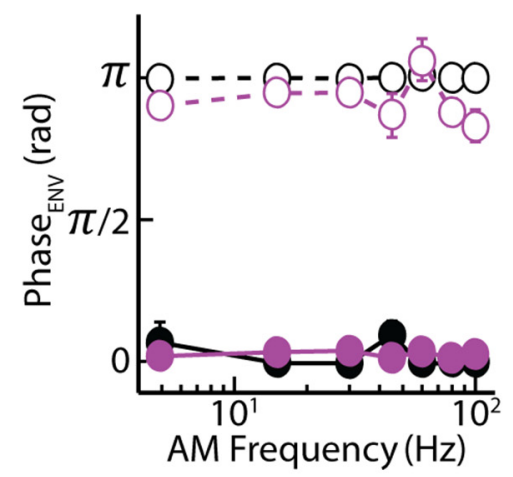

H

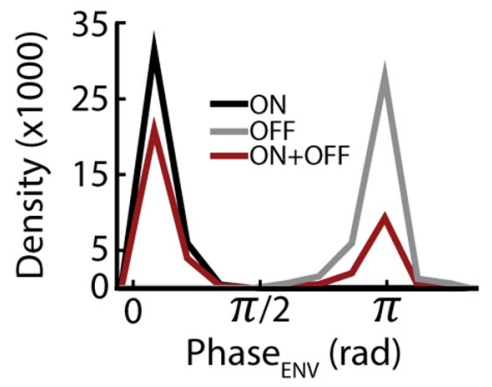

Figure 2. Two classes of peripheral single sensory neurons display ON-type and OFF-type responses to the envelope, respectively. $\boldsymbol{A}$, Example of an $0 \mathrm{~N}$-type $\mathrm{P}$-unit responding to an increase in envelope (top) with an increase in firing rate (middle) independently of the AM frequency content: black represents $5-15 \mathrm{~Hz}$; purple represents $60-80 \mathrm{~Hz}$. Bottom, Firing rate response to different envelope frequencies with time normalized to the envelope period. It is seen that the responses mostly superimpose. $\boldsymbol{B}$, Example of an OFF-type P-unit responding to an increase in envelope (top) with a decrease in firing rate (middle) independently

those elicited by the envelope: this is due to the fact that the firing rate modulations caused by the fast time-varying AM waveform then tend to average out partially over the longer timescale of the envelope. We however conclude that P-unit response heterogeneities to envelope (i.e., second-order) stimuli are not simply inherited from the observed largely homogeneous responses to AM (i.e., firstorder) stimuli.

\section{A cascade linear-nonlinear model of} $\mathrm{P}$-unit responses to $\mathrm{AMs}$ and envelopes To better understand how $\mathrm{P}$-units can simultaneously display homogeneous responses to AMs and heterogeneous responses to envelopes, we built a linear-nonlinear cascade model (Chichilnisky, 2001; Massot et al., 2012). This model, described in Figure $4 A$, consists of first linearly filtering the stimulus and adding a baseline value to form a linear prediction of the firing rate. This prediction is then passed through a static piecewise nonlinearity that takes into account both rectification (i.e., the firing rate cannot be negative by definition) and saturation (i.e., the firing rate cannot exceed a maximum value due to refractoriness) to obtain the predicted firing rate (Fig. 4A).

We first tested whether this model could accurately describe $\mathrm{P}$-unit responses to the

\footnotetext{
of the AM frequency content: black represents $5-15 \mathrm{~Hz}$; purple represents $60-80 \mathrm{~Hz}$. Bottom, Firing rate response to different envelope frequencies with time normalized to the envelope period. It is seen that the responses mostly superimpose. C, Population-averaged gain to the envelope as a function of envelope frequency for two different AM signals: black represents frequency range $5-15 \mathrm{~Hz}$; purple represents frequency range $60-80 \mathrm{~Hz}$ for $\mathrm{ON}$ (solid) and OFF (dashed) P-units. D, Population-averaged gain to the envelope as a function of average $\mathrm{AM}$ frequency for two different envelope frequencies: black represents $0.05 \mathrm{~Hz}$; purple represents $0.5 \mathrm{~Hz}$ for $\mathrm{ON}$ (solid) and OFF (dashed) P-units. E, Population-averaged phase to the envelope as a function of envelope frequency for two different AM signals: black represents frequency range $5-15 \mathrm{~Hz}$; purple represents frequency range $60-80 \mathrm{~Hz}$ for $\mathrm{ON}$ (solid) and OFF (dashed) P-units. F, Population-averaged phase to the envelope as a function of average AM frequency for two different envelope frequencies: black represents 0.05 $\mathrm{Hz}$; purple represents $0.5 \mathrm{~Hz}$ for $\mathrm{ON}$ (solid) and OFF (dashed) $P$-units. G, Distributions of gain to the envelope across stimuli for all (dark red), ON (black), and OFF (gray) P-units. There was no significant difference between the gain distributions obtained for ON and OFF P-units (Kolmogorov-Smirnov test, $p=0.34)$. $\boldsymbol{H}$, Distributions of phase to the envelope across stimuli for all (dark red), ON (black), and OFF (gray) P-units. The phase distribution for all P-units consists of two wellseparated modes (Hartigan's dip test, $p \ll 10^{-3}$ ). Further, the phase distributions obtained for $\mathrm{ON}$ and $\mathrm{OFF} \mathrm{P}$-units were significantly different (Kolmogorov-Smirnov test, $p \ll$ $\left.10^{-3}\right)$. Error bars indicate SEM.
} 
A

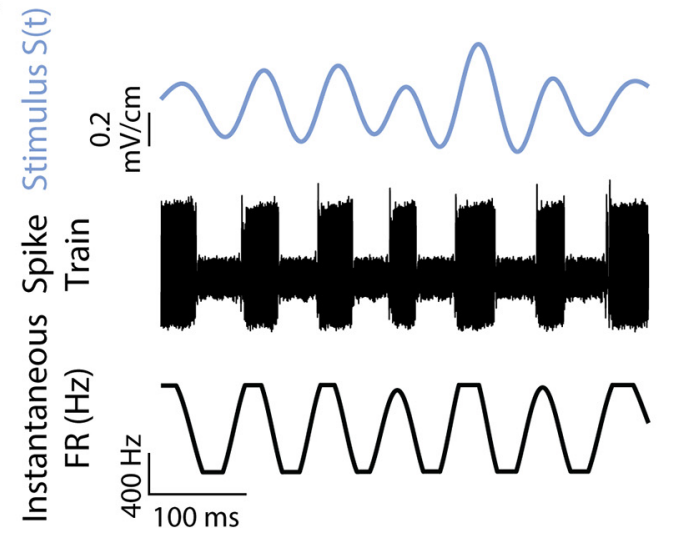

C

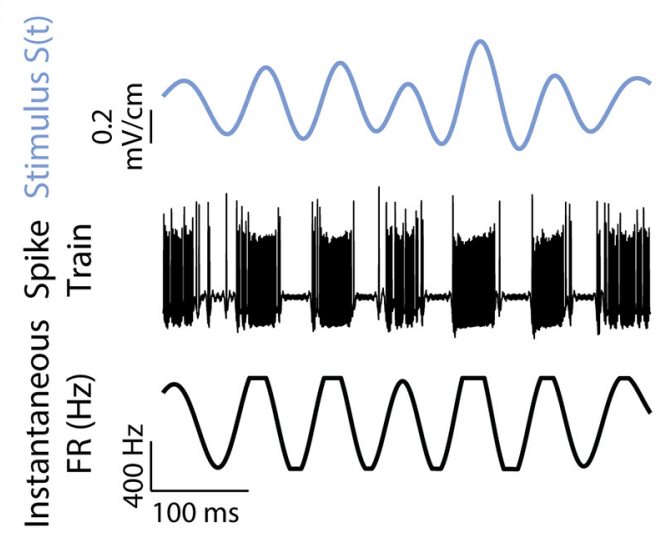

E

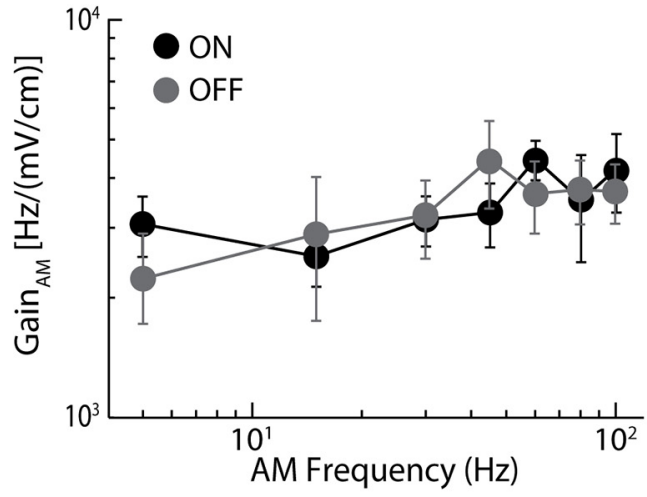

B

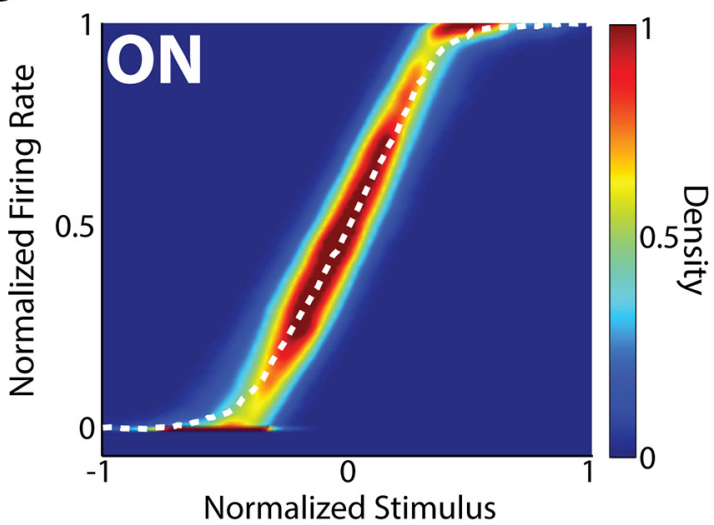

D

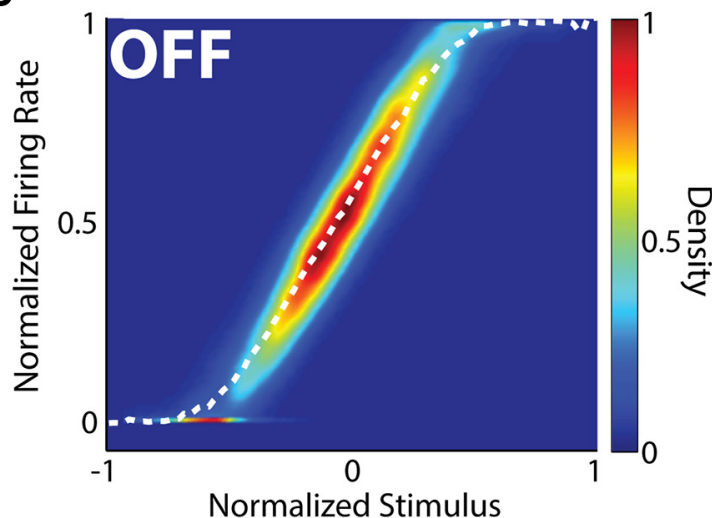

$\mathbf{F}$

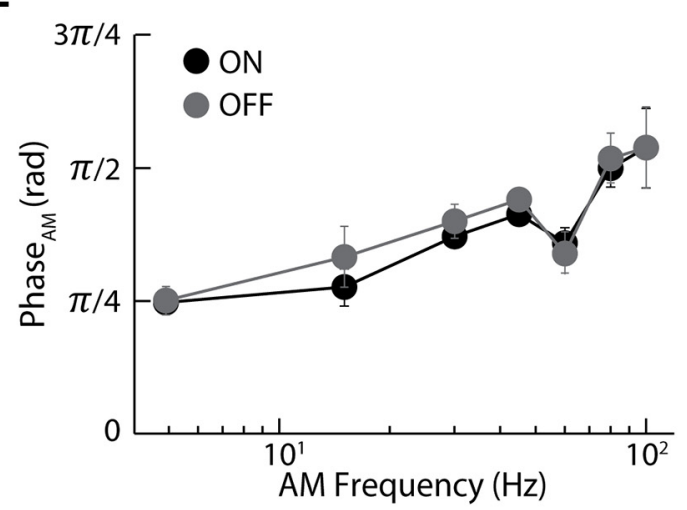

Figure 3. ON-type and OFF-type P-units display similar tuning curves to the stimulus. A, Example of an ON-type P-unit's time-dependent firing rate (bottom) to the stimulus (top). This unit had a baseline firing rate of $270 \mathrm{~Hz}$. The spike train (middle) displays phase-locking as spikes were only elicited in response to the large positive portions of the $60-80 \mathrm{~Hz}$ noise stimulus. $\boldsymbol{B}$, Normalized tuning curve (white dashed line) showing the normalized mean firing rate as well as the density of the firing rate (color plot) as a function of the normalized stimulus for the $0 \mathrm{~N}$-type $\mathrm{P}$-unit shown in $\boldsymbol{A}$. C, Example of an OFF-type P-unit's time-dependent firing rate (bottom) to the same stimulus (top) as in $\boldsymbol{A}$. The spike train (middle) displays phase-locking qualitatively similar to that of the ON-type P-unit shown in $\boldsymbol{A}$. This unit had a baseline firing rate of $416 \mathrm{~Hz}$. $\boldsymbol{D}$, Normalized tuning curve (white dashed line) showing the normalized mean firing rate as well as the firing rate density (color plot) as a function of the normalized stimulus for the OFF-type P-unit shown in C. E, Population-averaged gain values with respect to the stimulus for ON-type (black) and $0 \mathrm{FF}$ type (gray) $P$-units as a function of the average AM frequency. There were no significant differences between $0 \mathrm{~N}$ - and OFF-type P-units (two-way ANOVA, $p>0.08$ ). Higher-frequency AMs gave rise to higher gain values in general. $\boldsymbol{F}$, Population-averaged phase values with respect to the stimulus for $\mathrm{ON}$ (black) and $0 \mathrm{FF}$-type $\mathrm{P}$-units (gray) as function of average AM frequency. There were no significant differences between $\mathrm{ON}$ - and OFF-type P-units for any of the AMs used (two-way ANOVA, $p>0.06$ ). Higher-frequency AMs gave rise to higher phase leads in general. Error bars indicate SEM.

AM waveform. Our modeling predictions were accurate for both ON-type (Fig. 4B) and OFF-type (Fig. 4C) P-unit firing rate responses to AM stimuli. We analyzed the model's output in the same way as the experimental data and used linear-systems identification techniques to compute the gain and phase of the response to the AM. Overall, there was good agreement between the gain predicted from our model and that computed from experimental data across our dataset (Fig. 4D) and the populationaveraged gain values agreed for all AM stimuli tested (two-way
ANOVA, $p>0.23$ ) (Fig. 4E). There was also good agreement between the predicted phase of the response and the experimentally measured one (Fig. $4 F$ ) and the population-averaged phase values agreed for all AM stimuli tested (two-way ANOVA, $p>$ 0.13 ) (Fig. 4G). We conclude that P-unit responses to AMs can be explained based on their linear filtering properties, a static piecewise nonlinearity, and their baseline firing rates.

We next tested whether this simple model could also accurately describe $\mathrm{P}$-unit responses to envelopes. To do so, we used 
A

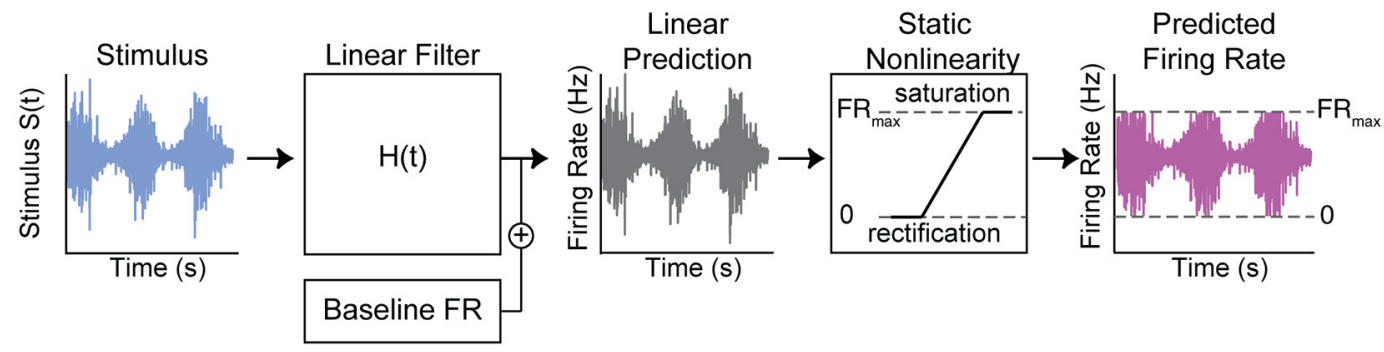

B

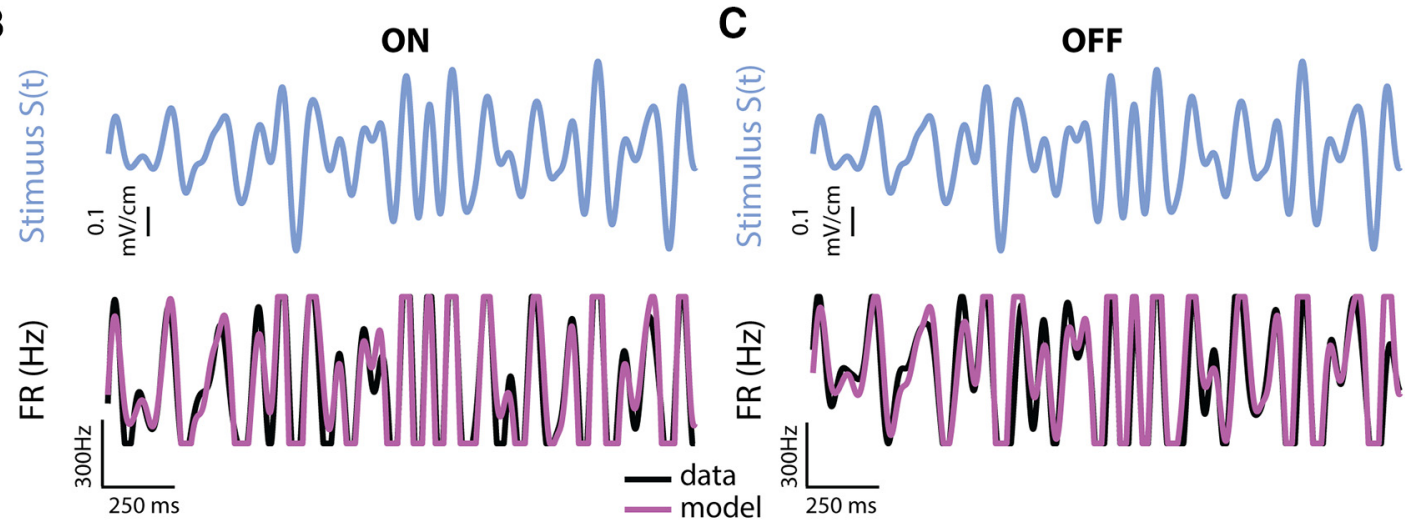

D

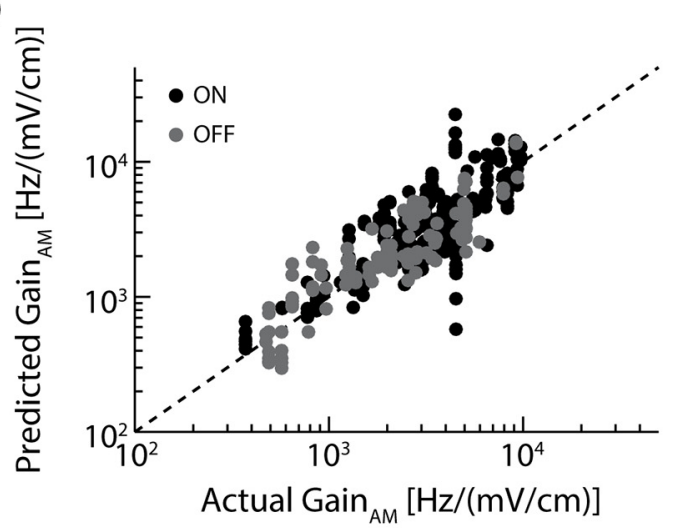

E

$\mathbf{F}$

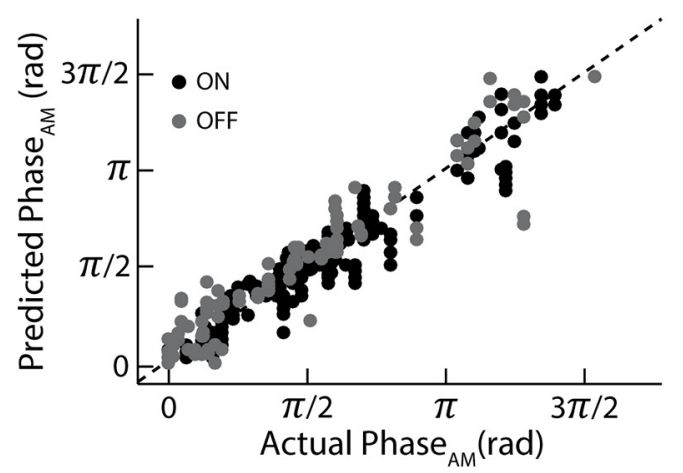

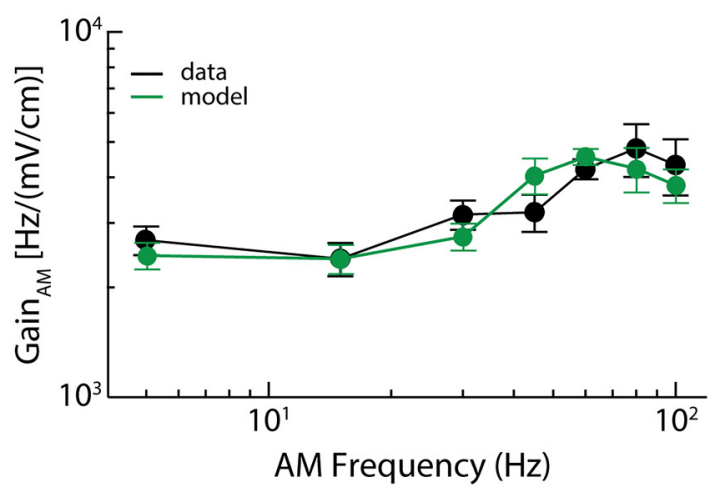

G

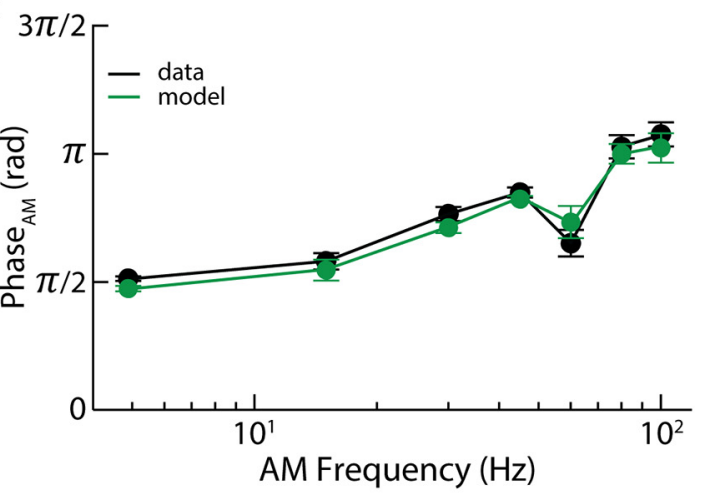

Figure 4. A linear-nonlinear (LN) cascade model predicts P-unit responses to AMs. A, Schematic showing the LN model's structure. The stimulus (left) is first convolved with a filter $H(t)$ that is determined from data to generate the linear predicted firing rate (middle). The baseline firing rate is then added to the linear prediction, and the resulting signal is passed through a static nonlinearity that takes into account rectification and saturation to obtain the predicted firing rate (right). $\boldsymbol{B}$, Traces showing our model's prediction (bottom, purple) with the actual response (bottom, black) to the AM (top, blue) for an example ON-type P-unit. C, Traces showing the model's prediction (bottom, purple) with the actual response (bottom, black) to the AM (top, blue) for an example OFF-type P-unit. $\boldsymbol{D}$, Predicted gain to the AM as a function of the actual gain to the AM for all ON-type (black) and OFF-type (gray) P-units in our dataset. The data points are scattered around the identity line (black dashed line). $\boldsymbol{E}$, Population-averaged gain as a function of the average AM frequency for our data (black) and our model's prediction (green). $\boldsymbol{F}$, Predicted phase to the AM from our model as a function of actual phase to the AM for all ON-type (black) and OFF-type (gray) P-units in our datasets. G, Population-averaged phase as a function of the average AM frequency for our data (black) and our model's prediction (green). Error bars indicate SEM. 

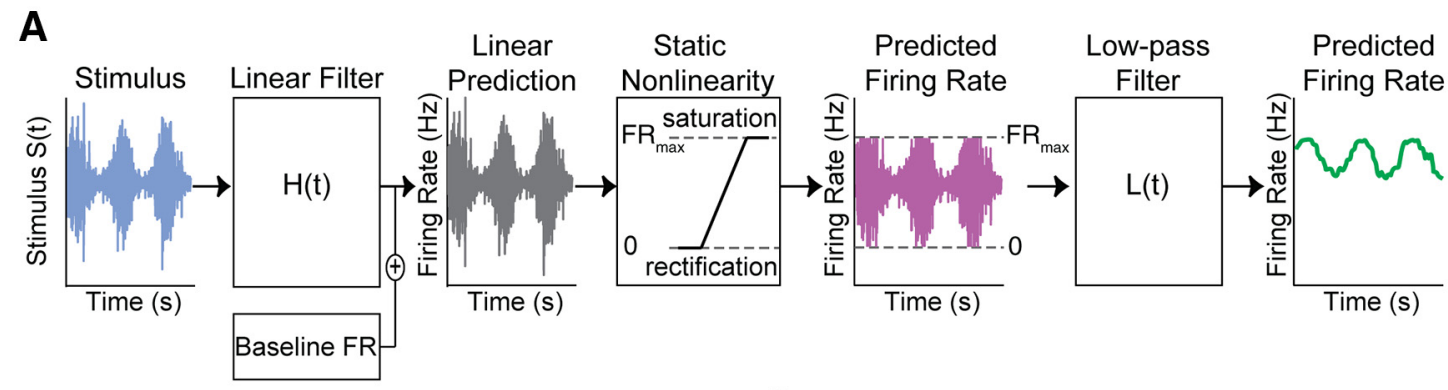

B

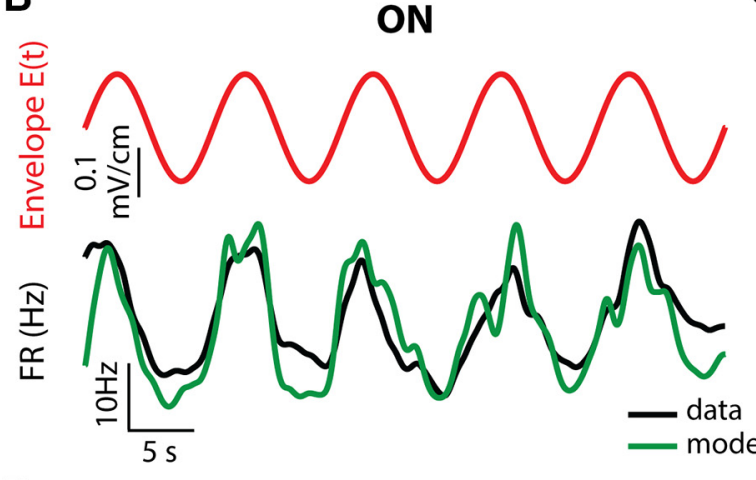

D

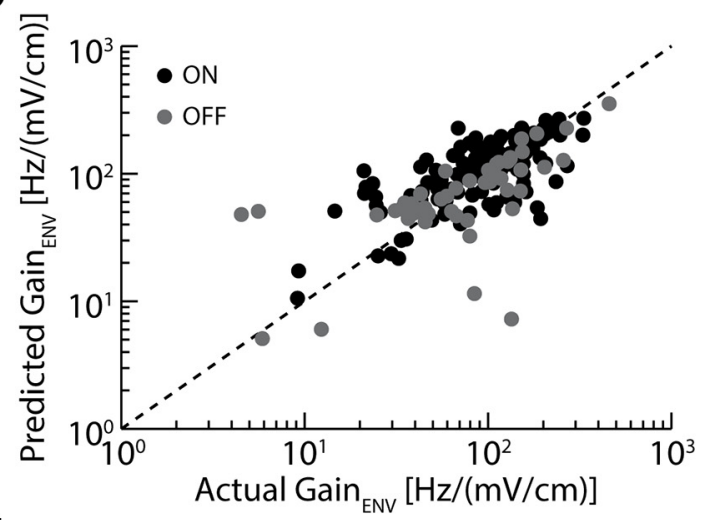

$\mathbf{F}$

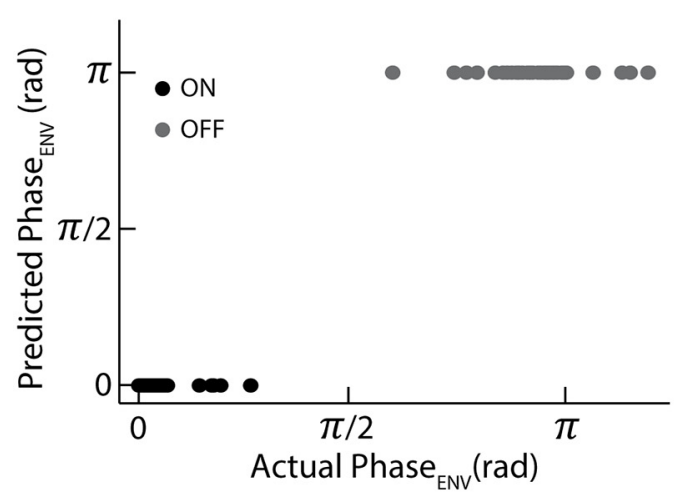

C

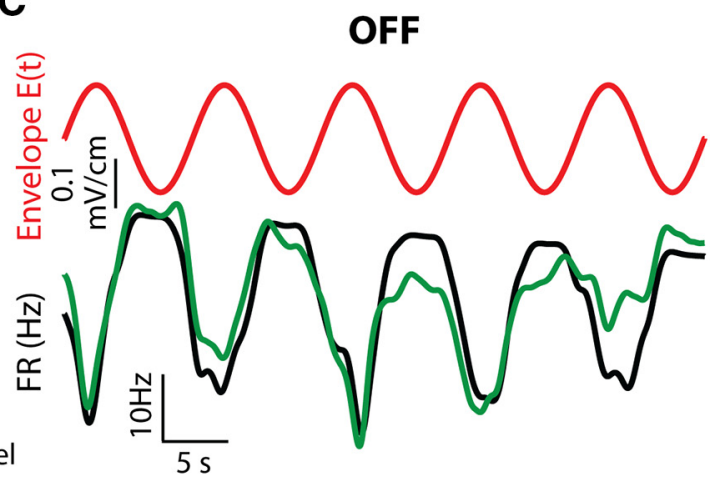

E

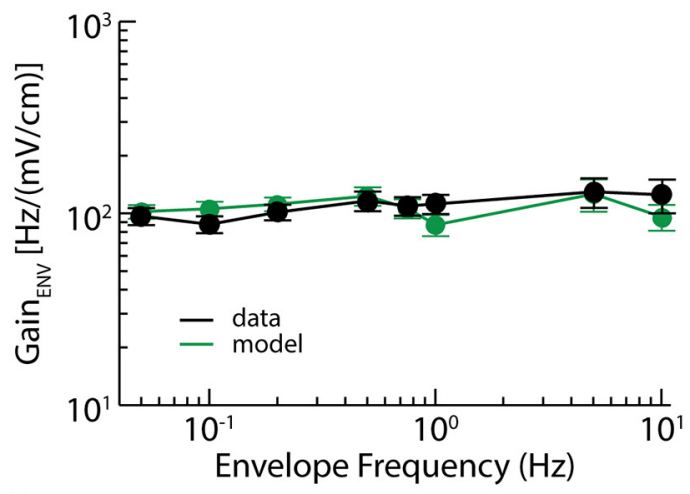

G

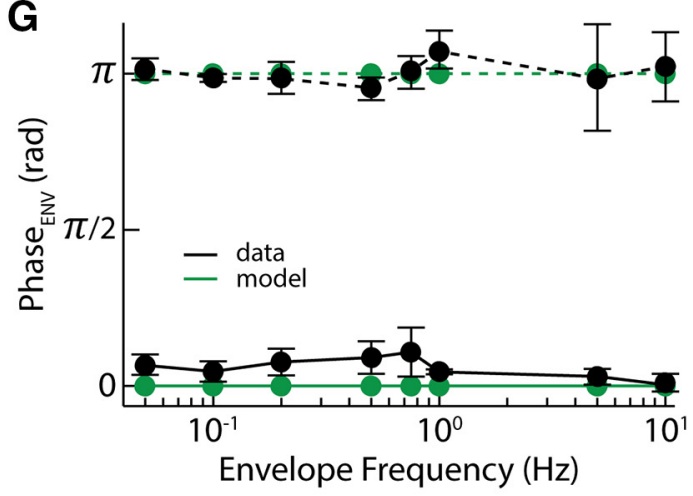

Figure 5. A linear-nonlinear (LN) cascade model predicts P-unit responses to envelopes. $A$, Schematic showing the LN model's structure. The stimulus (outside left) is first convolved with a filter $H(t)$ that is determined from data to generate the linear predicted firing rate (left). The baseline firing rate is then added to the linear prediction, and the resulting signal is passed through a static nonlinearity that takes into account rectification and saturation to obtain the predicted firing rate (right). The resulting signal is then low-pass filtered to obtain the predicted response to the envelope (outside right). $\boldsymbol{B}$, Traces showing our model's prediction (bottom, green) with the actual response (bottom, black) to the envelope (top, red) for an example 0N-type P-unit. $\boldsymbol{C}$, Traces showing the model's prediction (bottom, green) with the actual response (bottom, black) to the envelope (top, red) for an example OFF-type P-unit. $D$, Predicted gain to the envelope as a function of the actual gain to the envelope for all ON-type (black) and OFF-type (gray) P-units in our dataset. The data points are scattered around the identity line (dashed black line). $E$, Population-averaged gain as a function of envelope frequency for our data (black) and our model's prediction (green). $\boldsymbol{F}$, Predicted phase to the envelope from our model as a function of actual phase to the envelope for all ON-type (black) and OFF-type (gray) P-units in our dataset. G, Population-averaged phase as a function of envelope frequency for our data (black) and our model's prediction (green) for ON (bottom) and OFF (top) -type P-units. Error bars indicate SEM. 
A

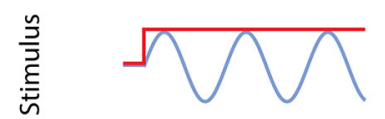

FR.

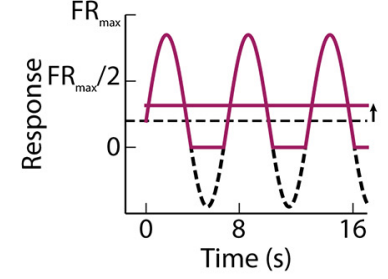

B

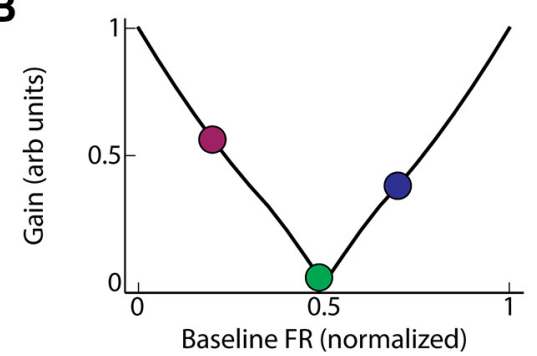

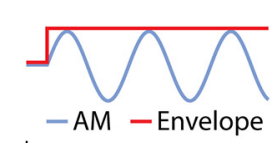
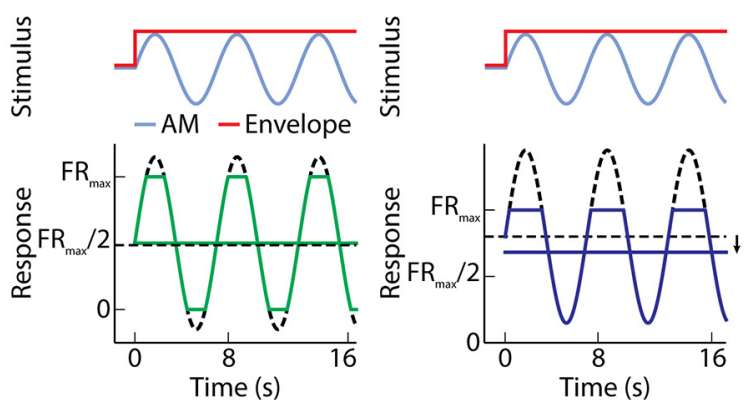

C

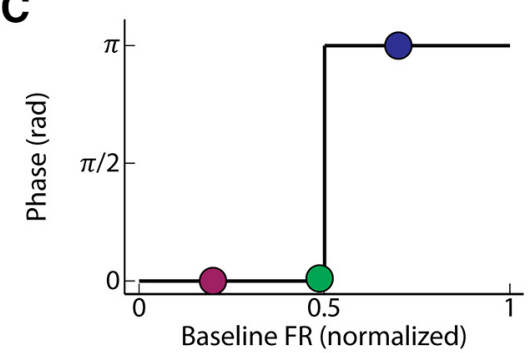

Figure 6. Model predicts baseline firing determines $0 \mathrm{~N}$ - vs OFF-type responses to envelopes. $\boldsymbol{A}$, Left, Schematic showing why a $\mathrm{P}$-unit with low baseline firing rate gives an $\mathrm{ON}$-type response (bottom, solid purple curve) to the envelope of a sinusoidal stimulus (top): because of rectification at zero, the mean firing rate increases (bottom, solid horizontal purple line) during stimulation compared with what it would be if no rectification occurred (dashed horizontal black line) as indicated by the arrow. Bottom, Dashed black curve indicates the response to the same stimulus without the static nonlinearity. Middle, Schematic showing why a P-unit with baseline firing rate in the middle of the dynamic range (i.e., equal to $\mathrm{FR}_{\max } / 2$ ) does not respond to the envelope. For this unit, the increase in firing rate due to rectification is exactly compensated by the decrease due to saturation (bottom, solid green curve). Thus, the mean firing rate (bottom, horizontal solid green line) is not different from the baseline firing rate (bottom, horizontal dashed black line). Bottom, Dashed black curve indicates the response to the same stimulus without the static nonlinearity. Right, Schematic showing why a P-unit with high baseline firing rate gives an OFF-type response (bottom, solid blue curve) to the envelope of a sinusoidal stimulus (top): because of saturation, the mean firing rate decreases (bottom, blue horizontal line) during stimulation compared with what it would be if no saturation occurred (dashed black horizontal line) as indicated by the arrow. Bottom, Dashed black curve indicates the response to the same stimulus without the static nonlinearity. $\boldsymbol{B}$, The gain displays a minimum when the normalized baseline firing rate (i.e., the baseline firing rate divided by the maximum firing rate obtained during stimulation) is equal to 0.5 . C, Phase of the response as a function of normalized baseline firing rate. Responses are 0N-type for normalized baseline firing rates $<0.5$ and OFF-type otherwise.

the same model as in Figure 4A, but low-pass filtered the output firing rate as done for the experimental data to investigate firing rate fluctuations on the envelope timescale (Fig. $5 \mathrm{~A}$ ). We found that our simple model could accurately predict both ON-type (Fig. 5B, green) and OFF-type (Fig. 5C, green) P-unit firing rate responses to envelopes. We analyzed the model's output in the same way as the experimental data and used linear-systems identification techniques to compute the gain and phase of the response to the envelope. Overall, there was good agreement between the gain predicted from our model and that computed from experimental data across our dataset (Fig. 5D), and the population-averaged gain values agreed for all envelope frequencies tested (two-way ANOVA, $p>0.17$ ) (Fig. 5E). There was also good agreement between the predicted phase of the response and the experimentally measured one (Fig. $5 F$ ), and the population-averaged phase values were in good agreement overall (Fig. 5G). We conclude that $\mathrm{P}$-unit responses to envelopes can be explained based on their linear filtering properties, a static piecewise nonlinearity, and their baseline firing rates.

Heterogeneous baseline firing properties determine gain and phase of the envelope response

We then used our model to generate predictions as to the mechanisms that underlie ON- and OFF-type responses to envelopes.
We focused on the baseline firing rate as well as the static nonlinearity because our previous results (Fig. 3) showed no major differences between the linear filtering properties of ON- and OFF-type P-units. To illustrate how both baseline firing rate and static nonlinearities can influence responses to envelopes, let us consider the three cases shown in Figure 6A and assume that the envelope varies slowly with respect to the AM (i.e., that the envelope does not vary much during one cycle of the AM and thus is effectively constant). In Figure 6A (left), a model neuron with low baseline firing rate chosen just above the zero rate level responds (Fig. 6A, bottom left, solid purple curve) in a linear fashion to the sinusoidal stimulus (Fig. $6 A$, top left), except for stimulus phases for which the firing rate is equal to zero (i.e., rectification). For comparison, the hypothetical firing rate response in the absence of rectification is also shown (Fig. $6 A$, left, dashed black curve). The mean of the firing rate response (Fig. 6A, left, solid horizontal purple line) is then offset positively with respect to its value in the absence of rectification (Fig. $6 A$, left, dashed horizontal black line). It is then easy to see that sinusoidal stimuli of larger amplitude will give rise to more rectification (i.e., the firing rate will be zero during a greater portion of the stimulus cycle), leading to a greater positive offset of the mean and that the response of this model neuron will thus be in phase with the envelope.

In contrast, Figure $6 \mathrm{~A}$ (right) shows the response of a neuron with a high baseline firing rate just below the maximum firing rate value $\mathrm{FR}_{\max }$. This neuron displays saturation in response to the sinusoidal stimulus (Fig. $6 \mathrm{~A}$, right, solid blue curve). Thus, the mean firing rate is then negatively offset (Fig. $6 A$, right, solid horizontal blue line) with respect to its value in the absence of saturation (Fig. 6A, right, dashed horizontal black line). Stimuli of larger amplitudes (i.e., envelopes) will elicit more saturation and thus a greater negative offset. The response of this model neuron will then be out of phase with the envelope. Figure $6 \mathrm{~A}$ (middle) shows the response of a neuron whose baseline firing rate is equal to $\mathrm{FR}_{\max } / 2$. The response of this neuron (Fig. $6 \mathrm{~A}$, middle, solid green curve) displays both rectification and saturation. However, the increase in firing rate due to rectification is then exactly compensated by the decrease in firing rate due to saturation. Thus, the mean firing rate (Fig. $6 \mathrm{~A}$, middle, solid green line) is equal to its value in the absence of stimulation (Fig. $6 \mathrm{~A}$, middle, dashed black line). This simple model thus predicts that the gain of the envelope response (i.e., the absolute change in firing rate divided by the stimulus amplitude) will be smallest for $\mathrm{P}$-units whose baseline firing rate is in the middle of the dynamic range (Fig. $6 \mathrm{~B}$ ) and that the phase is either zero for normalized baseline firing rates $<0.5$ and $180^{\circ}$ otherwise (Fig. $6 \mathrm{C}$ ). Thus, our model makes the important prediction that both the gain and the phase of the envelope response are determined by the baseline to maximum firing rate ratio for a given afferent. 


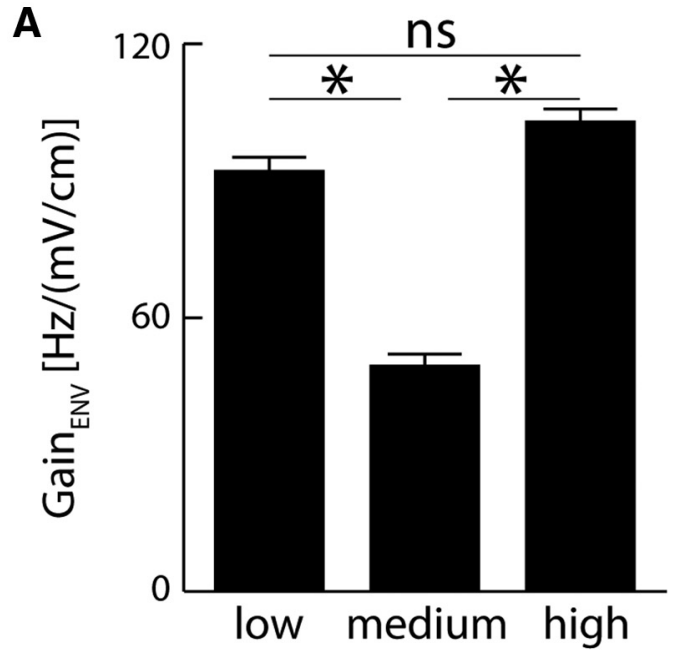

B

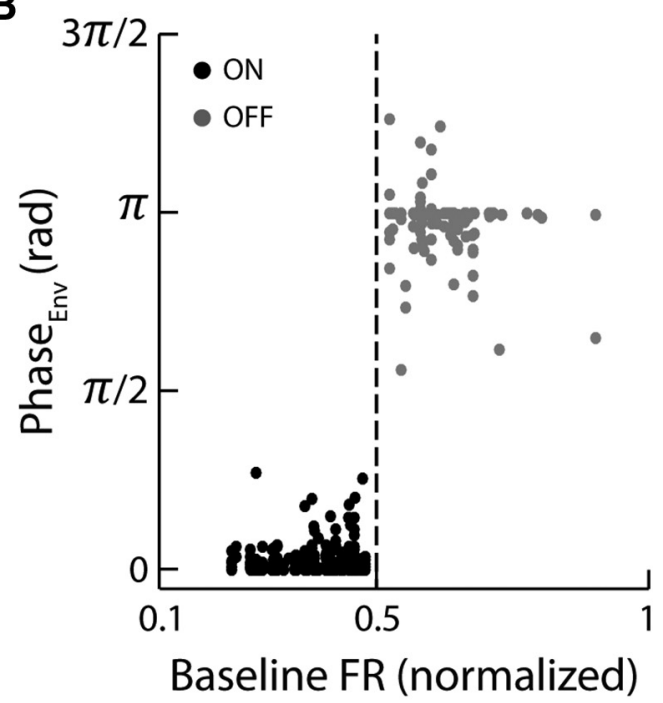

C

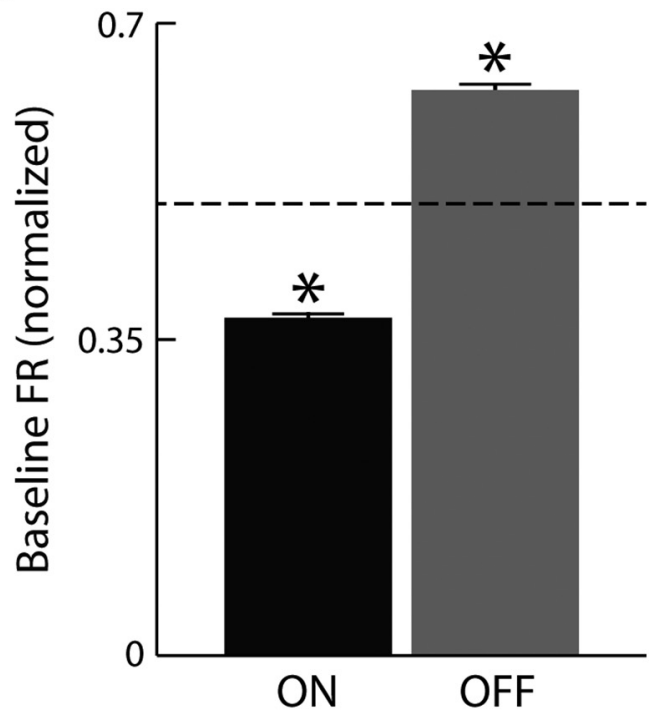

Figure 7. Verifying modeling predictions. $A$, Population-averaged gain values for $P$-units with low $(<0.48, N=72)$, medium $(>0.48$ and $<0.52, N=15)$, and high $(>0.52, N=49)$ normalized baseline firing rates. P-units with low and high normalized baseline firing rates displayed significantly larger gains than $P$-units with medium normalized baseline firing rates (two-way ANOVA, $p<0.004$ ). B, ON-type (black) responses are observed preferentially for $\mathrm{P}$-units with a normalized baseline firing rate $<0.5$, whereas OFF-type (gray) responses are
We verified our model's predictions with respect to gain experimentally by separating our recorded $\mathrm{P}$-units into three groups. We found that $\mathrm{P}$-units whose normalized baseline firing rates were both significantly higher than those whose normalized baseline firing rates were close to 0.5 (Fig. $7 \mathrm{~A}$, two-way ANOVA, $p<0.004)$. We next plotted the phase of the envelope response as a function of normalized baseline firing rate. Confirming our model's predictions, we found that most ON-type $(n=72,89 \%)$ and OFF-type $(n=49,89 \%)$ cells displayed normalized baseline firing rates that were $<0.5$ or $>0.5$, respectively (Fig. $7 B$ ). Further, the population-averaged normalized baseline firing rate of ON-type P-units was significantly $<0.5$ ( $t$ test, $n=72, p \ll$ $\left.10^{-3}\right)$, whereas that of OFF-type P-units was significantly $>0.5$ ( $t$ test, $n=49, p \ll 10^{-3}$ ) (Fig. 7C).

\section{Stimulus reconstruction and population coding}

Finally, we quantified how well one can reconstruct the envelope waveform based on the activity of $\mathrm{P}$-unit populations. In particular, we were interested whether the observed heterogeneous responses might help optimize information transmission. We used the optimal stimulus reconstruction technique in which each spike train is convolved with an optimal filter to estimate the stimulus waveform (Rieke et al., 1996; Gabbiani and Koch, 1998; Massot et al., 2011) (Fig. 8A). Overall, we found that the coding fraction (i.e., the fraction of the stimulus waveform that is correctly reconstructed) increases with increasing population size (Fig. 8B). However, we found that the quality of the reconstruction for the envelope signal was significantly lower than that of unmodulated noisy AM waveforms (Fig. 8B), this is expected because $\mathrm{P}$-units display much greater sensitivities to the AM waveform than to the envelope (Figs. $2 C, D$ and $3 E$ ). By extrapolating the curves obtained in Figure $8 B$, we estimated that $N=$ $324 \pm 57$ P-units would be necessary to obtain a quality of reconstruction for envelopes similar to that seen for AMs, which is $\sim 2 \%$ of the total P-units population $(15,000)$ available in each fish: the implications of this result are discussed further below. We next compared the quality of the reconstruction using populations of $\mathrm{P}$-units of only a given type (i.e., $\mathrm{ON}$ or $\mathrm{OFF}$ ) or a mixed population (50\% ON and 50\% OFF). Although the quality of the reconstruction improved for increasing population size for all three groups (Fig. 8C), considering a mixed population gave rise to significantly higher coding fraction values than considering populations of either ON or OFF-type P-units of the same size (two-way ANOVAs with Bonferroni post hoc correction, $p \ll$ $10^{-3}$ ) (Fig. 8C). Thus, although within each class heterogeneities improve the quality of the reconstruction as a function of increasing population size, heterogeneities between the two classes gives rise to further improvement: this is a generalization to the envelope case of the benefit of adding ON- and OFF-type responses to first-order stimuli for improved reconstruction (Gabbiani, 1996). The quality of the reconstruction improved for increasing AM frequencies (Fig. 8D) but was independent of envelope frequency for a given population size (Fig. $8 E$ ), indicating that the $\mathrm{P}$-unit population faithfully transmits information about envelopes over the entire frequency range tested.

$\leftarrow$

instead preferentially observed for $\mathrm{P}$-units with higher normalized baseline firing rates. $\boldsymbol{C}$, The normalized baseline firing rate of $0 \mathrm{~N}$-type $\mathrm{P}$-units was on average $(0.38 \pm 0.01)$ significantly less than 0.5 ( $t$ test, $\mathrm{n}_{0 \mathrm{~N}}=72, p \ll 10^{-3}$ ), whereas that of OFF-type $P$-units was on average $(0.57 \pm 0.01)$ significantly greater than $0.5\left(t\right.$ test, $\left.\mathrm{n}_{\mathrm{OFF}}=49, p \ll 10^{-3}\right)$. Horizontal dashed line indicates the threshold value of 0.5 . 
A

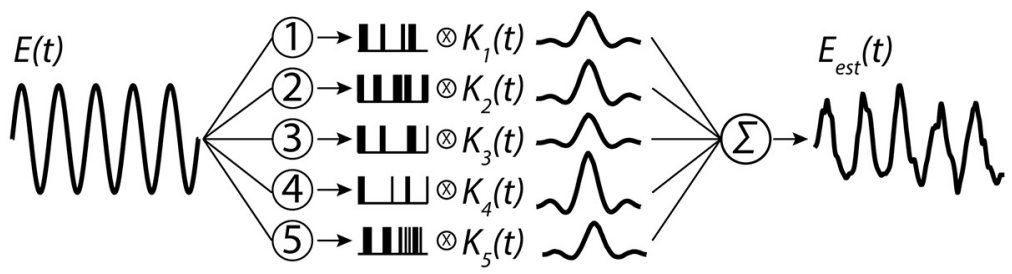

B

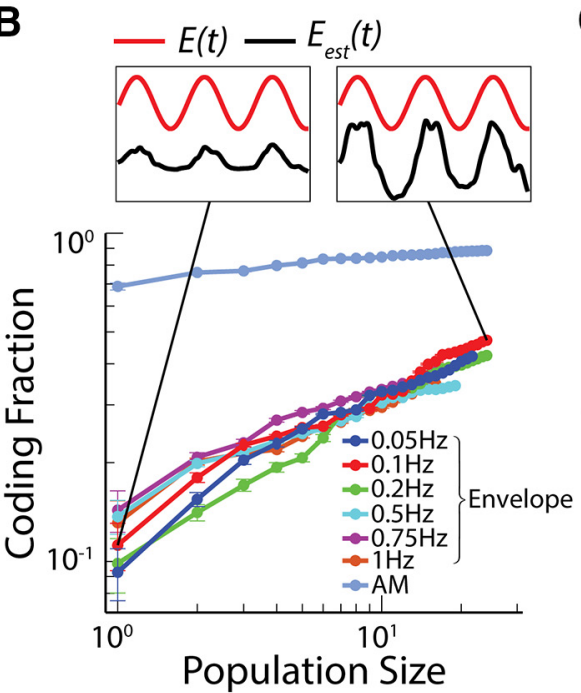

$\mathrm{C}$

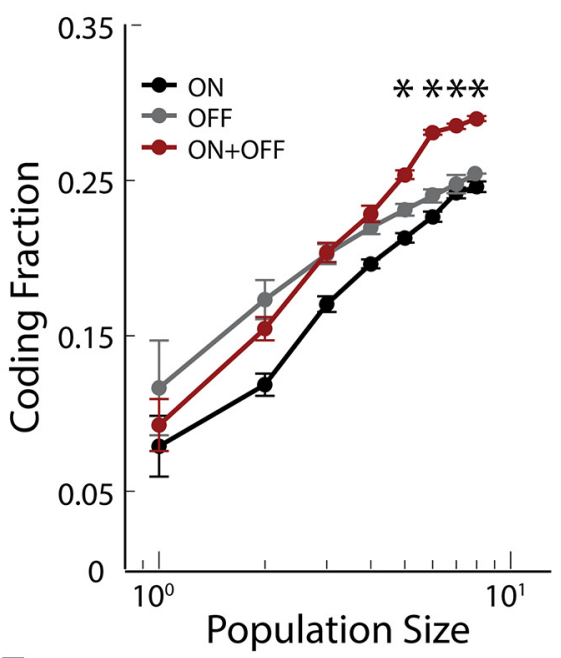

D

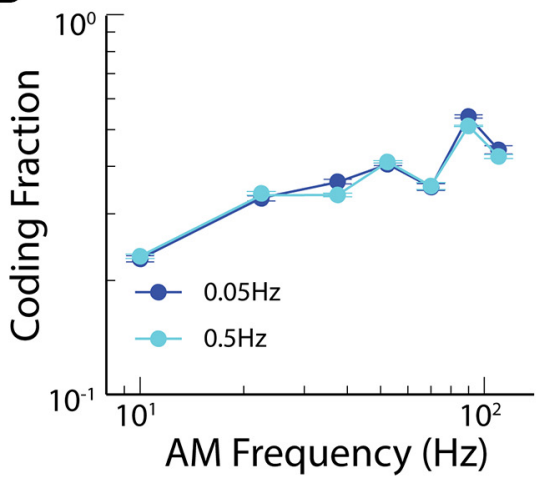

E

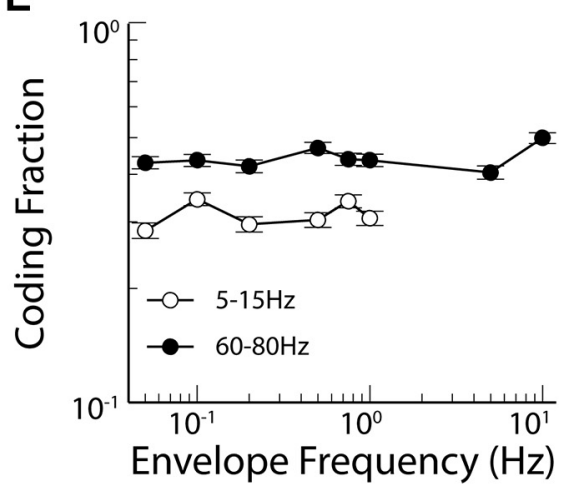

Figure 8. The P-unit population transmits detailed information about the envelope independently of envelope frequency. $\boldsymbol{A}$, Schematic of the stimulus reconstruction procedure. Each neural response $R_{i}(t)$ to the signal $E(t)($ left) was convolved $(\otimes)$ with an optimal filter $K_{i}(t)$ (middle). The results were then summed to get the estimated signal $E_{\text {est }}(t)$ (right). $\boldsymbol{B}$, Coding fraction (i.e., the fraction of the envelope that is successfully reconstructed) as a function of population size for all envelope frequencies using a 5-15 $\mathrm{Hz}$ AM stimulus. For comparison, the coding fraction quantifying the fraction of the unmodulated $5-15 \mathrm{~Hz}$ AM stimulus that is correctly reconstructed is also shown. Similar results were obtained using a 60-80 Hz AM stimulus (data not shown). C, Population-averaged coding fraction (i.e., the fraction of the envelope that is successfully reconstructed) as a function of population size for ON-type P-units (black), OFF-type P-units (gray), and a mixed population of ON + OFF-type P-units (dark red, 50\% ON and $50 \%$ OFF). The coding fraction for mixed population is significantly greater than that obtained when considering either $0 \mathrm{~N}$ - or OFF-type P-units for population $\geq 5 .{ }^{*} p=0.01$ (two-way ANOVA with Bonferroni post hoc correction). D, Population-averaged coding fraction for the envelope as a function of the average AM frequency for two envelope frequencies: dark blue represents 0.05 $\mathrm{Hz}$; light blue represents $0.5 \mathrm{~Hz}$. The coding fraction increased as a function of AM frequency and both curves were not significantly different from one another ( $t$ test, $p=0.7492$ ). $\boldsymbol{E}$, Population-averaged coding fraction obtained for the envelope as a function of envelope frequency for $5-15 \mathrm{~Hz}$ (open circles) and $60-80 \mathrm{~Hz}$ (filled circles) using a mixed population of $n=6 \mathrm{P}$-units.

\section{Discussion}

Summary of results

We studied the peripheral electroreceptor afferent responses to stimuli consisting of a fast time-varying waveform whose envelope varied sinusoidally. Analysis revealed two P-unit classes (ONand OFF-type) with similar sensitivities. Both classes displayed similar piecewise linear tuning curves to unmodulated first-order stimuli. To explain these results, we built a linear-nonlinear cascade model that incorporated known properties of P-units: namely, baseline firing rate, linear filtering properties, and a static piecewise nonlinearity. This model successfully explained variability in our dataset. Further analysis revealed that the normalized baseline firing rate determined the phase of the envelope response. We validated this prediction experimentally. Finally, we quantified the quality of the linear reconstruction of the envelope by P-units. The observed heterogeneous responses to envelopes (i.e., $\mathrm{ON}$ vs OFF-type) were advantageous as a heterogeneous population of $\mathrm{ON}$ and $\mathrm{OFF}$ $\mathrm{P}$-units transmitted more information than a population of more homogeneous $\mathrm{ON}$ or OFF P-units.

\section{Coding of envelopes in the electrosensory system}

Envelopes are a behaviorally relevant feature of electrosensory stimuli as they carry information about the relative positions between conspecifics as well as their identities (Yu et al., 2012; Fotowat et al., 2013; for review, see Stamper et al., 2013). In particular, envelopes can arise during two behaviorally relevant situations: (1) during movement between two conspecifics; and (2) from the static interactions between the EODs of three of more fish. Whereas the former movement envelopes generally contain low $(<1 \mathrm{~Hz})$ temporal frequencies (Yu et al., 2012; Fotowat et al., 2013; Metzen and Chacron, 2014), the latter "social" envelopes instead contain higher $(>1 \mathrm{~Hz})$ temporal frequencies (Stamper et al., 2010; Fotowat et al., 2013). Weakly electric fish perceive both movement and social envelopes as evidenced from behavioral responses (Stamper et al., 2012; Metzen and Chacron, 2014). Although it is known that electrosensory neurons, including $\mathrm{P}$-units, can respond to envelopes, all previous electrophysiological studies have used stimuli that mimicked social envelopes (Middleton et al., 2006; Savard et al., 2011; Vonderschen and Chacron, 2011; McGillivray et al., 2012), whereas we specifically considered stimuli that instead mimic movement envelopes. Our study is thus the first that investigates how electrosensory neurons respond to mimics of movement envelopes. Although our electrophysiological results have shown that the quality of envelope reconstruction obtained by combining P-unit spike trains was independent of frequency, our present results (Fig. 1D) as well as previous ones have shown that behavioral responses decreased as a function of envelope frequency (Metzen and Chacron, 2014). As such, behavioral responses are likely due to further processing of the detailed envelope information carried in $\mathrm{P}$-unit spike trains by 
downstream neurons. Further studies should thus focus on how downstream neurons decode envelope information. In particular, P-units synapse onto pyramidal neurons within three parallel maps of the body surface in the ELL with different response profiles to first-order features (Shumway, 1989; Krahe et al., 2008; Maler, 2009a, b; Khosravi-Hashemi and Chacron, 2014; Krahe and Maler, 2014), and future studies should focus on characterizing how pyramidal neurons, which are the sole output neurons of the ELL, respond to social and movement envelopes in a mapspecific manner.

Moreover, we note that we considered stimuli whose intensity was large enough to elicit nonlinear responses to individual $\mathrm{P}$-units. Stimuli with weaker intensities will elicit firing rate modulations from P-units that are linearly related to the stimulus (Gussin et al., 2007), and it is then necessary to perform a nonlinear transformation to extract the envelope (Middleton et al., 2007). Previous studies have shown that downstream targets of P-units, namely, pyramidal neurons as well as inhibitory interneurons, can respond to envelopes by performing nonlinear transformations (Middleton et al., 2006; McGillivray et al., 2012). However, whether and, if so, how these nonlinear transformations determine the phase of ELL pyramidal neuron responses to envelopes span the large dynamic range observed under natural conditions (Yu et al., 2012; Fotowat et al., 2013; Metzen and Chacron, 2014) have not been investigated to date. Further studies are therefore needed to investigate this important issue.

\section{Coding of first- and second-order features of natural stimuli by the sensory periphery}

Heterogeneous responses in peripheral sensory neural populations have been observed ubiquitously across systems and species and are frequently associated with distinct receptor types (e.g., Type I and II hair cells in the mammalian auditory and vestibular systems) (Hargrave and McDowell, 1992; Köppl, 1997; Goldberg, 2000; Gold and Gebhart, 2010; Eatock and Songer, 2011). This is however not the case for P-units (Zakon, 1987; Bennett et al., 1989).

What advantage is there then in having P-units display strong heterogeneities in their responses to envelopes (i.e., second-order features) while, at the same time, display weak heterogeneities in their responses to EOD AMs (i.e., first-order features)? We hypothesize that this is a consequence of the fact that $\mathrm{P}$-units, being peripheral sensory neurons, must optimize their coding properties to provide downstream neurons with the relevant information about both first- and second-order features of electrosensory stimuli that are largely independent of one another (Stamper et al., 2013; Metzen and Chacron, 2014) as seen in other systems (Simoncelli and Olshausen, 2001). The first-order features of natural electrosensory stimuli display large variations in their spatial extent as those caused by prey are spatially localized, whereas those caused by conspecifics are instead spatially diffuse (Zupanc and Maler, 1993; Nelson and MacIver, 1999). In contrast, second-order features of natural electrosensory stimuli only occur during interactions with conspecifics and thus will activate most, if not all, of the P-unit population (Stamper et al., 2013). We propose that the relatively homogeneous responses of $\mathrm{P}$-units to first-order features might help better signal the presence of the weak spatially localized stimuli caused by prey, as it would be theoretically easier to detect the resulting similar changes in activity across a small neural subpopulation. In contrast, as second-order features will activate most, if not all, of the $\mathrm{P}$-unit population, heterogeneities in their response instead allow for increased information transmission. It is conceivable that tradeoffs be- tween homogeneity and heterogeneity help optimize information transmission about both first- and second-order features of electrosensory stimuli, but further studies are needed to test this hypothesis.

\section{Role of neural heterogeneities in coding}

Neural heterogeneities have been found ubiquitously in the CNS. Although theoretical studies have shown that heterogeneous neural networks transmit more information than homogeneous ones (Mejias and Longtin, 2012), which is supported by experimental work (Marsat and Maler, 2010; Padmanabhan and Urban, 2010; Tripathy et al., 2013), how neural heterogeneities determine different response profiles to a given stimulus feature is poorly understood. Although cells with ON- and OFF-type responses have been reported across brain areas, these responses are typically mediated by direct excitation and inhibition through local interneurons (Saunders and Bastian, 1984; Imig et al., 1990; Ris and Godaux, 1998; Schiller, 2010). Our results showing that baseline firing rate, combined with static nonlinearities, can give rise to either ON- or OFF-type responses to envelopes thus provide the first evidence that a neural population with homogeneous tuning properties to one stimulus feature can display heterogeneous response profiles to another feature.

\section{Applicability to other systems}

Envelopes are a common feature of natural stimuli across systems, whose statistics are qualitatively similar to that considered here, which carry behaviorally relevant information and are essential for perception (Baker, 1999; Heil, 2003). However, comparatively little is known about the coding strategies used by the brain to process them (but for the auditory system, see Joris et al., 2004). Neurons displaying ON- and OFF-type responses to second-order features are likely to be found elsewhere. This is because neurons with high baseline firing rates can be found not only at the sensory periphery (Köppl, 1997; Goldberg, 2000) but also in more central brain areas (Kuffler, 1953; Rospars et al., 1994; Linden et al., 2009; Luczak et al., 2009). Moreover, many neurons display relative refractoriness (Häusser and Roth, 1997; Mickus et al., 1999; Chacron et al., 2001; Sadeghi et al., 2007) that, if elicited before rectification, will lead to OFF-type envelope responses. Although only a few studies have reported response phase to envelopes (Joris and Yin, 1992; Joris, 1996; Delgutte et al., 1997), the mechanisms reported here could explain why the responses of some auditory neurons are mostly in phase with the envelope whereas those of others are mostly out of phase $(\mathrm{Ku}-$ wada et al., 2014). In particular, although our results show that taking into account the known linear filtering properties of electroreceptor afferents and implementing static nonlinearities in the firing rate such as rectification and saturation is sufficient to account for their responses to envelopes, it is likely that other types of nonlinearities seen in other systems will also contribute to shaping responses to envelopes (French and Wong, 1977; Patuzzi and Robertson, 1988; Massot et al., 2012; Rho and Prescott, 2012). Further studies are however needed to test these predictions.

\section{References}

Avila-Åkerberg O, Krahe R, Chacron MJ (2010) Neural heterogeneities and stimulus properties affect burst coding in vivo. Neuroscience 168:300313. CrossRef Medline

Baker CL Jr (1999) Central neural mechanisms for detecting second-order motion. Curr Opin Neurobiol 9:461-466. CrossRef Medline

Bannister NJ, Larkman AU (1995a) Dendritic morphology of CA1 pyrami- 
dal neurones from the rat hippocampus: II. Spine distributions. J Comp Neurol 360:161-171. CrossRef Medline

Bannister NJ, Larkman AU (1995b) Dendritic morphology of CA1 pyramidal neurones from the rat hippocampus: I. Branching patterns. J Comp Neurol 360:150-160. CrossRef Medline

Bastian J (1981) Electrolocation II: the effects of moving objects and other electrical stimuli on the activities of two categories of posterior lateral line lobe cells in Apteronotus albifrons. J Comp Physiol A 144:481-494. CrossRef

Bastian J (1996a) Plasticity in an electrosensory system: II. Postsynaptic events associated with a dynamic sensory filter. J Neurophysiol 76:24972507. Medline

Bastian J (1996b) Plasticity in an electrosensory system: I. General features of a dynamic sensory filter. J Neurophysiol 76:2483-2496. Medline

Bastian J, Chacron MJ, Maler L (2002) Receptive field organization determines pyramidal cell stimulus-encoding capability and spatial stimulus selectivity. J Neurosci 22:4577-4590. Medline

Bennett MV, Sandri C, Akert K (1989) Fine structure of the tuberous electroreceptor of the high-frequency electric fish, Sternachus albifrons (gymnotiformes). J Neurocytol 18:265-283. CrossRef Medline

Chacron MJ, Bastian J (2008) Population coding by electrosensory neurons. J Neurophysiol 99:1825-1835. CrossRef Medline

Chacron MJ, Longtin A, Maler L (2001) Negative interspike interval correlations increase the neuronal capacity for encoding time-varying stimuli. J Neurosci 21:5328-5343. Medline

Chacron MJ, Doiron B, Maler L, Longtin A, Bastian J (2003) Non-classical receptive field mediates switch in a sensory neuron's frequency tuning. Nature 423:77-81. CrossRef Medline

Chacron MJ, Maler L, Bastian J (2005) Electroreceptor neuron dynamics shape information transmission. Nat Neurosci 8:673-678. CrossRef Medline

Chacron MJ, Longtin A, Maler L (2011) Efficient computation via sparse coding in electrosensory neural networks. Curr Opin Neurobiol 21:752760. CrossRef Medline

Cherif S, Cullen KE, Galiana HL (2008) An improved method for the estimation of firing rate dynamics using an optimal digital filter. J Neurosci Methods 173:165-181. CrossRef Medline

Chichilnisky EJ (2001) A simple white noise analysis of neuronal light responses. Network 12:199-213. CrossRef Medline

Dan Y, Alonso JM, Usrey WM, Reid RC (1998) Coding of visual information by precisely correlated spikes in the lateral geniculate nucleus. Nat Neurosci 1:501-507. CrossRef Medline

Dayan P, Abbott LF (2001) Theoretical neuroscience: computational and mathematical modeling of neural systems. Cambridge, MA: Massachusetts Institute of Technology.

Delgutte B, Hammond BM, Cariani PA (1997) Neural coding of the temporal envelope of speech: relation to modulation transfer functions. In: Psychophysical and physiological advances in hearing (Palmer AR, Rees A, Summerfield AQ, Meddis R, eds), pp 595-603. London: Whurr.

Eatock RA, Songer JE (2011) Vestibular hair cells and afferents: two channels for head motion signals. Annu Rev Neurosci 34:501-534. CrossRef Medline

Fotowat H, Harrison RR, Krahe R (2013) Statistics of the electrosensory input in the freely swimming weakly electric fish Apteronotus leptorhynchus. J Neurosci 33:13758-13772. CrossRef Medline

French AS, Wong RK (1977) Nonlinear analysis of sensory transduction in an insect mechanoreceptor. Biol Cybern 26:231-240. CrossRef Medline

Gabbiani F (1996) Coding of time varying signals in spike trains of linear and half-wave rectifying neurons. Network 7:61-85. CrossRef

Gabbiani F, Koch C (1998) Principles of spike train analysis. In: Methods in neuronal modeling: from ions to networks (Koch C, Segev I, eds), pp 313-360. Cambridge, MA: Massachusetts Institute of Technology.

Gabbiani F, Metzner W, Wessel R, Koch C (1996) From stimulus encoding to feature extraction in weakly electric fish. Nature 384:564-567. CrossRef Medline

Gold MS, Gebhart GF (2010) Nociceptor sensitization in pain pathogenesis. Nat Med 16:1248-1257. CrossRef Medline

Goldberg JM (2000) Afferent diversity and the organisation of central vestibular pathways. Exp Brain Res 130:277-297. CrossRef Medline

Gussin D, Benda J, Maler L (2007) Limits of linear rate coding of dynamic stimuli by electroreceptor afferents. J Neurophysiol 97:2917-2929. CrossRef Medline
Hargrave PA, McDowell JH (1992) Rhodopsin and phototransduction. Int Rev Cytol 137B:49-97. Medline

Häusser M, Roth A (1997) Dendritic and somatic glutamate receptor channels in rat cerebellar Purkinje cells. J Physiol 501:77-95. CrossRef Medline

Heil P (2003) Coding of temporal onset envelope in the auditory system. Speech Commun 41:123-134. CrossRef

Hitschfeld ÉM, Stamper SA, Vonderschen K, Fortune ES, Chacron M) (2009) Effects of restraint and immobilization on electrosensory behaviors of weakly electric fish. ILAR J 50:361-372. CrossRef Medline

Imig TJ, Irons WA, Samson FR (1990) Single-unit selectivity to azimuthal direction and sound pressure level of noise bursts in cat high-frequency primary auditory cortex. J Neurophysiol 63:1448-1466. Medline

Joris PX (1996) Envelope coding in the lateral superior olive: II. Characteristic delays and comparison with responses in the medial superior olive. J Neurophysiol 76:2137-2156. Medline

Joris PX, Yin TC (1992) Responses to amplitude-modulated tones in the auditory nerve of the cat. J Acoust Soc Am 91:215-232. CrossRef Medline

Joris PX, Schreiner CE, Rees A (2004) Neural processing of amplitudemodulated sounds. Physiol Rev 84:541-577. CrossRef Medline

Khosravi-Hashemi N, Chacron MJ (2014) Motion processing across multiple topographic maps in the electrosensory system. Physiol Rep 2:e00253. CrossRef Medline

Köppl C (1997) Frequency tuning and spontaneous activity in the auditory nerve and cochlear nucleus magnocellularis of the barn owl Tyto alba. J Neurophysiol 77:364-377. Medline

Krahe R, Maler L (2014) Neural maps in the electrosensory system of weakly electric fish. Curr Opin Neurobiol 24:13-21. CrossRef Medline

Krahe R, Kreiman G, Gabbiani F, Koch C, Metzner W (2002) Stimulus encoding and feature extraction by multiple sensory neurons. J Neurosci 22:2374-2382. Medline

Krahe R, Bastian J, Chacron MJ (2008) Temporal processing across multiple topographic maps in the electrosensory system. J Neurophysiol 100:852867. CrossRef Medline

Kuffler SW (1953) Discharge patterns and functional organization of mammalian retina. J Neurophysiol 16:37-68. Medline

Kuwada S, Bishop B, Kim DO (2014) Azimuth and envelope coding in the inferior colliculus of the unanesthetized rabbit: effect of reverberation and distance. J Neurophysiol 112:1340-1355. CrossRef Medline

Linden ML, Heynen AJ, Haslinger RH, Bear MF (2009) Thalamic activity that drives visual cortical plasticity. Nat Neurosci 12:390-392. CrossRef Medline

Luczak A, Barthó P, Harris KD (2009) Spontaneous events outline the realm of possible sensory responses in neocortical populations. Neuron 62:413425. CrossRef Medline

Lundstrom BN, Fairhall AL, Maravall M (2010) Multiple timescale encoding of slowly varying whisker stimulus envelope in cortical and thalamic neurons in vivo. J Neurosci 30:5071-5077. CrossRef Medline

Maler L (2009a) Receptive field organization across multiple electrosensory maps: I. Columnar organization and estimation of receptive field size. J Comp Neurol 516:376-393. CrossRef Medline

Maler L (2009b) Receptive field organization across multiple electrosensory maps: II. Computational analysis of the effects of receptive field size on prey localization. J Comp Neurol 516:394-422. CrossRef Medline

Marder E, Goaillard JM (2006) Variability, compensation and homeostasis in neuron and network function. Nat Rev Neurosci 7:563-574. CrossRef Medline

Márquez BT, Krahe R, Chacron MJ (2013) Neuromodulation of early electrosensory processing in gymnotiform weakly electric fish. J Exp Biol 216:2442-2450. CrossRef Medline

Marsat G, Maler L (2010) Neural heterogeneity and efficient population codes for communication signals. J Neurophysiol 104:2543-2555. CrossRef Medline

Marsat G, Longtin A, Maler L (2012) Cellular and circuit properties supporting different sensory coding strategies in electric fish and other systems. Curr Opin Neurobiol 22:686-692. CrossRef Medline

Massot C, Chacron MJ, Cullen KE (2011) Information transmission and detection thresholds in the vestibular nuclei: single neurons versus population encoding. J Neurophysiol 105:1798-1814. CrossRef Medline

Massot C, Schneider AD, Chacron MJ, Cullen KE (2012) The vestibular system implements a linear-nonlinear transformation in order to encode self-motion. PLoS Biol 10:e1001365. CrossRef Medline

McGillivray P, Vonderschen K, Fortune ES, Chacron MJ (2012) Parallel 
coding of first- and second-order stimulus attributes by midbrain electrosensory neurons. J Neurosci 32:5510-5524. CrossRef Medline

Mejias JF, Longtin A (2012) Optimal heterogeneity for coding in spiking neural networks. Phys Rev Lett 108:228102. CrossRef Medline

Metzen MG, Chacron MJ (2014) Weakly electric fish display behavioral responses to envelopes naturally occurring during movement: implications for neural processing. J Exp Biol 217:1381-1391. CrossRef Medline

Mickus T, Jung Hy, Spruston N (1999) Properties of slow cumulative sodium channel inactivation in rat hippocampal CA1 pyramidal neurons. Biophys J 76:846-860. CrossRef Medline

Middleton JW, Longtin A, Benda J, Maler L (2006) The cellular basis for parallel neural transmission of a high-frequency stimulus and its low-frequency envelope. Proc Natl Acad Sci U S A 103:14596-14601. CrossRef Medline

Middleton JW, Harvey-Girard E, Maler L, Longtin A (2007) Envelope gating and noise shaping in populations of noisy neurons. Phys Rev E Stat Nonlin Soft Matter Phys 75:021918. CrossRef Medline

Myers LJ, Lowery M, O’Malley M, Vaughan CL, Heneghan C, St Clair Gibson A, Harley YX, Sreenivasan R (2003) Rectification and non-linear preprocessing of EMG signals for cortico-muscular analysis. J Neurosci Methods 124:157-165. CrossRef Medline

Nelson ME, Maciver MA (1999) Prey capture in the weakly electric fish Apteronotus albifrons: sensory acquisition strategies and electrosensory consequences. J Exp Biol 202:1195-1203. Medline

Nelson ME, Xu Z, Payne JR (1997) Characterization and modeling of P-type electrosensory afferent responses to amplitude modulations in a wavetype electric fish. J Comp Physiol A 181:532-544. CrossRef Medline

Padmanabhan K, Urban NN (2010) Intrinsic biophysical diversity decorrelates neuronal firing while increasing information content. Nat Neurosci 13:1276-1282. CrossRef Medline

Patuzzi R, Robertson D (1988) Tuning in the mammalian cochlea. Physiol Rev 68:1009-1082. Medline

Rho YA, Prescott SA (2012) Identification of molecular pathologies sufficient to cause neuropathic excitability in primary somatosensory afferents using dynamical systems theory. PLoS Comput Biol 8:e1002524. CrossRef Medline

Rieke F, Warland D, de Ruyter van Steveninck RR, Bialek W (1996) Spikes: exploring the neural code. Cambridge, MA: Massachusetts Institute of Technology.

Ris L, Godaux E (1998) Neuronal activity in the vestibular nuclei after contralateral or bilateral labyrinthectomy in the alert guinea pig. J Neurophysiol 80:2352-2367. Medline

Rospars JP, Lánský P, Vaillant J, Duchamp-Viret P, Duchamp A (1994) Spontaneous activity of first- and second-order neurons in the frog olfactory system. Brain Res 662:31-44. CrossRef Medline

Sadeghi SG, Minor LB, Cullen KE (2007) Response of vestibular-nerve afferents to active and passive rotations under normal conditions and after unilateral labyrinthectomy. J Neurophysiol 97:1503-1514. Medline

Saunders J, Bastian J (1984) The physiology and morphology of two classes of electrosensory neurons in the weakly electric fish Apteronotus leptorhynchus. J Comp Physiol A 154:199-209. CrossRef

Savard M, Krahe R, Chacron MJ (2011) Neural heterogeneities influence envelope and temporal coding at the sensory periphery. Neuroscience 172:270-284. CrossRef Medline

Scheich H, Bullock TH, Hamstra RH Jr (1973) Coding properties of two classes of afferent nerve fibers: high frequency electroreceptors in the electric fish, Eigenmania. J Neurophysiol 36:39-60. Medline
Schiller PH (2010) Parallel information processing channels created in the retina. Proc Natl Acad Sci U S A 107:17087-17094. CrossRef Medline

Schulz DJ, Goaillard JM, Marder E (2006) Variable channel expression in identified single and electrically coupled neurons in different animals. Nat Neurosci 9:356-362. CrossRef Medline

Shumway CA (1989) Multiple electrosensory maps in the medulla of weakly electric Gymnotiform fish: I. Physiological differences. J Neurosci 9:43884399. Medline

Simmonds B, Chacron MJ (2015) Activation of parallel fiber feedback by spatially diffuse stimuli simultaneously reduces signal and noise correlations via independent mechanisms in a cerebellum-like structure. PLoS Comp Biol 11:e1004034. CrossRef Medline

Simoncelli EP, Olshausen BA (2001) Natural image statistics and neural representation. Annu Rev Neurosci 24:1193-1216. CrossRef Medline

Stamper SA, Carrera GE, Tan EW, Fugère V, Krahe R, Fortune ES (2010) Species differences in group size and electrosensory interference in weakly electric fishes: implications for electrosensory processing. Behav Brain Res 207:368-376. CrossRef Medline

Stamper SA, Madhav MS, Cowan NJ, Fortune ES (2012) Beyond the Jamming Avoidance Response: weakly electric fish respond to the envelope of social electrosensory signals. J Exp Biol 215:4196-4207. CrossRef Medline

Stamper SA, Fortune ES, Chacron MJ (2013) Perception and coding of envelopes in weakly electric fishes. J Exp Biol 216:2393-2402. CrossRef Medline

Toporikova N, Chacron MJ (2009) Dendritic SK channels gate information processing in vivo by regulating an intrinsic bursting mechanism seen in vitro. J Neurophysiol 102:2273-2287. CrossRef Medline

Tripathy SJ, Padmanabhan K, Gerkin RC, Urban NN (2013) Intermediate intrinsic diversity enhances neural population coding. Proc Natl Acad Sci U S A 110:8248-8253. CrossRef Medline

Vonderschen K, Chacron MJ (2011) Sparse and dense coding of natural stimuli by distinct midbrain neuron subpopulations. J Neurophysiol 106: 3102-3118. CrossRef Medline

Warland DK, Reinagel P, Meister M (1997) Decoding visual information from a population of retinal ganglion cells. J Neurophysiol 78:2336-2350. Medline

Xu Z, Payne JR, Nelson ME (1996) Logarithmic time course of sensory adaptation in electrosensory afferent nerve fibers in a weakly electric fish. J Neurophysiol 76:2020-2032. Medline

Yu N, Hupe GJ, Garfinkle C, Lewis JE, Longtin A (2012) Coding conspecific identity and motion in the electric sense. PLoS Comp Biol 8:e1002564. CrossRef Medline

Zakon HH (1987) Variation in the mode of receptor cell addition in the electrosensory system of Gymnotiform fish. J Comp Neurol 262:195-214. CrossRef Medline

Zakon HH, Oestreich J, Tallarovic S, Triefenbach F (2002) EOD modulations of brown ghost electric fish: JARs, chirps, rises, and dips. J Physiol (Paris) 96:451-458. CrossRef Medline

Zeng FG, Nie K, Stickney GS, Kong YY, Vongphoe M, Bhargave A, Weit C, Cao K (2005) Speech recognition with amplitude and frequency modulations. Proc Natl Acad Sci U S A 102:2293-2298. CrossRef Medline

Zohary E, Shadlen MN, Newsome WT (1994) Correlated neuronal discharge rate and its implications for psychophysical performance. Nature 370:140-143. CrossRef Medline

Zupanc GKH, Maler L (1993) Evoked chirping in the weakly electric fish Apteronotus leptorhynchus: a quantitative biophysical analysis. Can J Zool 71:2301-2310. CrossRef 